\title{
Modeling chemical reactions in porous media: a review
}

Received: 8 September 2020 / Accepted: 27 August 2021 / Published online: 14 September 2021

(C) The Author(s) 2021

\begin{abstract}
First, different porous media theories are presented. Some approaches are based on the classical mixture theory for fluids introduced in the 1960s by Truesdell and Coworkers. One of the first researchers who extended the theory to porous media (thus mixtures containing at least one solid constituent) and also accounting for chemical reactions was Bowen. Another important branch of porous media theory goes back to Biot. In the beginning, he dealt with classical geotechnical problems and set up his model empirically. Mathematicians often use reaction-diffusion equations which are limited in comparison with continuum models by several restrictive assumptions and very often only applicable to special problems. In this paper, the focus lies on approaches based on the mixture theory which incorporate chemical reactions. Different strategies to describe the chemical potential for mixtures are presented, and different opinions about the exploitation of the second law of thermodynamics for mixtures are put forward. Finally, several works of different types including chemical reactions in porous media are summarized.
\end{abstract}

Keywords Chemical reactions $\cdot$ Mixture theory $\cdot$ Porous media

\section{Introduction}

The protection and conservation of cultural goods from natural stones, as, for example, historical monuments made of sandstone, subject to weathering, can happen by filling the pores by a water-lime mixture. As illustrated in Fig. 1a, the mixture penetrates into the near surface pore structure of the stone and the calcium hydroxide reacts with carbon dioxide and builds calcium carbonate and water. Thus, a solid layer is built in the pore space which strengthens the bonds lost due to weathering. For results of an experimental simulation of the process with corresponding pictures, see, e.g., [50]. One real example is the Königin-Victoria-Denkmal (Queen Victoria Memorial) in the wineyards of Hochheim in Germany which was build in 1854 in memory of the visit of the British queen Victoria regarding her journey along the Rhine river in 1845. In 2019, the heavily weathered monument was under reconstruction. The conservation has been finished in 2020 so that the visit of the queen 175 years ago could be celebrated with the tudor turret in old splendor.

A second example where chemical reactions appear in porous media is permeable reactive barriers. As illustrated in Fig. 1b, these are constructions built of permeable reactive materials either in the ground or in flowing waters which are created in the flow paths of contaminated (ground)water. Due to reactive processes between the pollutants in the water and the material of the retention barrier, a remediation of the groundwater takes place. For an overview on developments of the last centuries, see [95].

Communicated by Andreas Öchsner.

B. Detmann $(\varangle)$

Universität Duisburg-Essen, Essen, Germany

E-mail: bettina.detmann@uni-due.de 


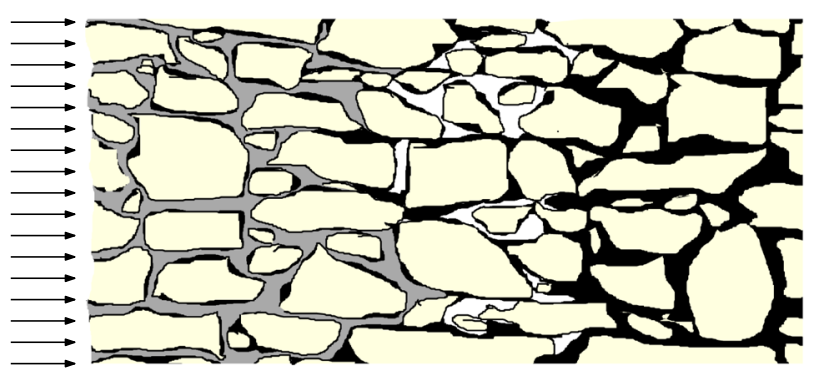

(a)

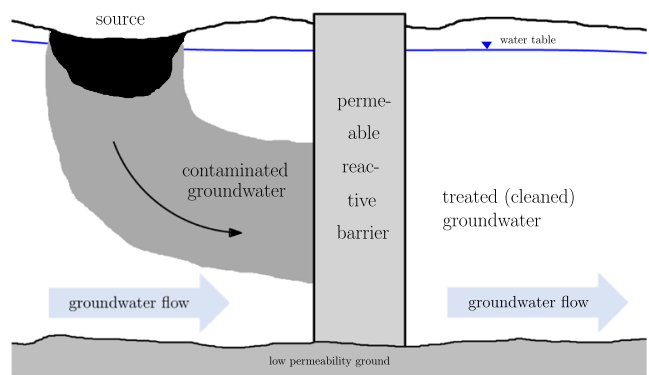

(b)

Fig. 1 a Conservation of weathered stonework. On the right, deep inside the stonework, the original filling of the pores (shown in black) is still present. From the left, where the stonework is strongly weathered and the channels between the particles are only filled sporadically with the original material, a water-lime mixture is brought into the pores. There the calcium hydroxide reacts with carbon dioxide. The new filling of the pores (in gray) strengthens the stonework. In the middle, there remain channels which the mixture cannot reach. This happens not only because the channels are dead-ended but also because the penetration depth of the mixture is limited. The pores remain unfilled (white). b Schematic illustration of a permeable reactive barrier which is built in the flow path of contaminated groundwater. In the barrier, either physical adsorption or reactive processes take place which lead to a remediation of the groundwater (color figure online)

It is worth mentioning that in various fields examples of reactive porous media occur. In biology, they appear, e.g., in tissues, bioreactors and biofilms, and in classical chemical and petroleum engineering, e.g., in packed bed reactors or oil recovery with miscible fluids.

These are examples of processes and materials where chemical reactions in porous media take place. This article aims to summarize different approaches which were intended to describe such or similar processes by models which stem from different backgrounds. It must be pointed out that not physical processes like adsorption are of interest but processes which lead to the chemical transformation of one set of chemical substances to another. Adsorption-diffusion processes have been studied by ${ }^{1}$ B. ALBERS, e.g., in [1] or [3].

\section{Overview on porous media theories}

One branch of the porous media theory has developed from the mixture theory (see Subsection 2.1) introduced by C.A. TRUESDELL in the 1960s [120]. In [35], R.M. BowEN initially investigated the mixture theory and extended it subsequently to porous media [36,37]. He also was one of the first researchers who dealt with chemically reacting mixtures $[33,34]$ even though at that time subject of consideration was miscible mixtures of fluids and not immiscible mixtures mostly considered in porous media theories. The development of thermodynamic mixture theories started by the discoveries of A. FICK [56] who postulated a linear relation between the diffusion flux and the concentration gradient. This is known as Fick's law of diffusion, and it is analogous to other relationships discovered at the same epoch which became important in the meantime as, for example, Darcy's law of hydraulic flow (will be discussed later), Ohm's law of charge transport or Fourier's law of heat transfer. Already in 1871, J. STEFAN [117] generalized Fick's law to more than two constituents. Starting with C. ECKART [54], when linear irreversible thermodynamics was created, Fick's law was generalized and the diffusion flux was considered to be a linear combination of the gradients of temperature and chemical potentials (see [93]). Although working rather well for many applications this form of constitutive relation for the diffusion flux leads to a parabolic diffusion equation and thereby predicts infinite speeds of propagation of disturbances in concentration. This is known as the paradox of diffusion and shown in [93]. As pointed out in [14] by R.J. ATKIN and R.E. CRAINE due to successive revisions of ideas and a certain element of controversy, the subsequent literature on the developments of the mixture theory is not easy to follow. However, hereinafter it is tried to summarize the most important developments, especially those which are important for the description of chemical reactions within continuum thermodynamic theories. For a general overview, it is referred to [14] where both chemically reacting and non-chemically reacting continua are considered or [13] where mixtures of fluids, mixtures of solids and mixtures of an elastic solid and a viscous fluid are discussed. Furthermore, many classical textbooks on thermodynamics, as, for example, DE Groot/MaZur [47], Gurtin et al. [67], MUller [93], PASSMAn et al. [99], Prigogine [102], RaAts [103],

\footnotetext{
1 The author married in 2016 and changed her name from Albers to Detmann.
} 
SilHaVy [114], SLATtery et al. [115], TRUESDELl [119] or WoOdS [131], provide a thermodynamically rigorous framework for the understanding how reactive mixtures should be modeled on the continuum level.

It should be mentioned that even for problems without chemical reactions difficulties regarding the application of mixture theories to porous media have been addressed, e.g., by W. NoLL who already in the abstract of [96] notes that for him even the notion mixture theory is misleading, and thereupon, he classifies the large literature on the subject as mostly defective. Works by R. SAMPAIO, M.E. GURTIN, M.L. OLIVER and especially W.O. Williams (see [124] and references therein) he considers sound but still lacking a precise conceptual foundation. The just mentioned researchers worked in the aftermath on the improvement in the theory, especially on the elimination of problems as, for example, the formulation of the stress tensor in mixtures. For the same purpose, subsequently, also the Thermodynamically Constrained Averaging Theory by W.G. GRAY and C.T. MILLER has been developed [64,65]. In [64], also chemical reactions are addressed.

Another important branch of porous media theory goes back to M.A. ВІот. In the beginning, he dealt with classical geotechnical problems like consolidation [27], afterward-mainly intended for the investigation of elastic wave propagation-he extended his considerations to the description of two-component porous media, i.e., fluid saturated solids [28]. Later on, BIOT investigated also chemical reactions [26].

For a historical review of the formulation of porous media theories, see, e.g., DE BOER and EHLERs [46].

\subsection{Approaches on the basis of mixture theory}

In soil mechanics, classically simple one-component models are used to describe processes in soils, rocks, and sediments. However, if the relative motion of a fluid through the channels of the solid cannot be neglected, such models fail and the consequence was the development of multi-phase theories considering bodies existing of fluid and at least one solid component. It was obvious to rely on a theory which had been developed for a mixture of a number of components before, namely the mixture theory for fluids. In the following, this theory is shortly revisited.

1. Classical mixture theory (fluid mixtures)

TRUESDELL introduced a continuous description of a body consisting of a different fluid components in Eulerian description. Each of the components has an own velocity field:

$$
\mathbf{v}^{\alpha}=\mathbf{v}^{\alpha}(\mathbf{x}, t), \quad \alpha=1, \ldots, \mathrm{A}, \quad \mathbf{x} \in \mathcal{B}_{t}, \quad t \in \mathfrak{T} .
$$

The position vector $\mathbf{x}$ specifies a position in the current configuration $\mathcal{B}_{t}$, and $t$ is an instant of time in the time domain $T$.

Therewith, for each component partial balance equations can be specified (see, e.g., TRUESDELL and TOUPIN [120]).

$$
\begin{array}{ll}
\text { mass } & \frac{\partial \rho^{\alpha}}{\partial t}+\operatorname{div} \rho^{\alpha} \mathbf{v}^{\alpha}=\hat{\rho}^{\alpha}, \\
\text { momentum } & \frac{\partial \rho^{\alpha} \mathbf{v}^{\alpha}}{\partial t}+\operatorname{div}\left(\rho^{\alpha} \mathbf{v}^{\alpha} \otimes \mathbf{v}^{\alpha}-\mathbf{T}^{\alpha}\right)=\hat{\mathbf{p}}_{t}^{\alpha}+\rho^{\alpha} \mathbf{b}^{\alpha}, \\
\text { energy } & \frac{\partial}{\partial t}\left[\rho^{\alpha}\left(\varepsilon^{\alpha}+\frac{1}{2} v^{\alpha 2}\right)\right]+\operatorname{div}\left[\rho^{\alpha}\left(\varepsilon^{\alpha}+\frac{1}{2} v^{\alpha 2}\right) \mathbf{v}^{\alpha}\right. \\
& \left.+\mathbf{q}^{\alpha}-\mathbf{T}^{\alpha} \mathbf{v}^{\alpha}\right]=\hat{\varepsilon}^{\alpha}+\rho^{\alpha} \mathbf{b}^{\alpha} \cdot \mathbf{v}^{\alpha}+\rho^{\alpha} r^{\alpha} .
\end{array}
$$

Therein, the following notation is used:

$\rho^{\alpha} \quad$ - partial mass density of component $\alpha$ per unit volume of the current configuration (cc)

$\hat{\rho}^{\alpha} \quad$ - mass source of component $\alpha$ per unit volume of cc

$\mathbf{T}^{\alpha} \quad$ - partial Cauchy stress tensor of component $\alpha$

$\hat{\mathbf{p}}_{t}^{\alpha} \quad$ - partial momentum source of component $\alpha$

$\mathbf{b}^{\alpha} \quad$ - partial volume force of component $\alpha$ per unit mass of cc

$\varepsilon^{\alpha} \quad$ - partial inner energy of component $\alpha$ per unit mass of cc

$\mathbf{q}^{\alpha} \quad$ - partial heat flux of component $\alpha$ per unit surface of cc

$\hat{\varepsilon}^{\alpha} \quad$ - partial energy source of component $\alpha$ per unit volume of cc

$r^{\alpha} \quad$ - partial energy radiation of component $\alpha$ per unit mass of cc.

Models for multi-component bodies are a better approximation to reality than classical models for onecomponent bodies. However, both classes of models describe with different accuracy the same processes. 
This means that each for mass, momentum and energy the sum of equations has to correspond to the respective balance equations for a one-component body. This yields constraints for the source terms and contributions describing the influence of the different kinematics on the process in the one-component model.

A comparison of the classical (single-component) and the partial balance equations for a multi-component mixture yields:

$$
\begin{aligned}
\rho & \equiv \sum_{\alpha=1}^{\mathrm{A}} \rho^{\alpha}, \quad \rho \mathbf{v} \equiv \sum_{\alpha=1}^{\mathrm{A}} \rho^{\alpha} \mathbf{v}^{\alpha}, \\
\sum_{\alpha=1}^{\mathrm{A}} \hat{\rho}^{\alpha} & =0, \quad \sum_{\alpha=1}^{\mathrm{A}} \hat{\mathbf{p}}_{t}^{\alpha}=0, \quad \sum_{\alpha=1}^{\mathrm{A}} \hat{\varepsilon}^{\alpha}=0 .
\end{aligned}
$$

Thus, for the stress tensor it follows:

$$
\mathbf{T}=\underbrace{\sum_{\alpha=1}^{\mathrm{A}} \mathbf{T}^{\alpha}}_{\text {static part }}+\underbrace{\frac{1}{\sum_{\alpha=1}^{\mathrm{A}} \rho_{t}^{\alpha}}\left(\sum_{\alpha=1}^{\mathrm{A}} \rho_{t}^{\alpha} \mathbf{v}^{\alpha}\right) \otimes\left(\sum_{\alpha=1}^{\mathrm{A}} \rho_{t}^{\alpha} \mathbf{v}^{\alpha}\right)-\sum_{\alpha=1}^{\mathrm{A}}\left(\rho_{t}^{\alpha} \mathbf{v}^{\alpha} \otimes \mathbf{v}^{\alpha}\right)}_{\text {kinematic part }} .
$$

In the one-component model, in which the stress tensor $\mathbf{T}$ is defined, a material surface moves with the velocity $\mathbf{v}$ defined by the second identity of (5). However, if we have a multi-component body, partial velocities of the particular constituents appear. In a two-component body consisting of a solid and a fluid, the commonly much lower velocity of the solid $\mathbf{v}^{S}$ and the velocity of the fluid $\mathbf{v}^{F}$ occur. Thus, single points have the velocity $\mathbf{v}^{\alpha}$ of the certain constituent and not the velocity $\mathbf{v}$ of the one-component body. A relative movement between points of the different constituents appears by which they transport a certain amount of momentum. This yields the kinematic part of the stress tensor. Similar contributions are obtained for heat flux and energy which contains the kinematic energy of relative motion.

For an overview on porous media model formulations by the theory of mixtures, see R.M. BowEN [38]. However, in this book chapter, for the sake of brevity, chemical reactions and other types of mass exchanges have been omitted from all models. All the same the reference [38] is very helpful because it is explained how continuum mechanical models are constructed: model formulations follow an almost standard development. First one proposes constitutive equations which define the particular model. These constitutive equations are then examined for possible restrictions implied by the entropy inequality and invariance requirements. Invariance requirements under changes of frame are called the axiom of material frame indifference and hold for all models. Invariance requirements under changes of reference configuration are called material symmetry restrictions and are special requirements of particular models. Interested readers are referred to Section 5.2 of B. ALBERS [1] (unfortunately in German) where the derivation of a continuum model for adsorption-diffusion processes from thermodynamic principles is shown in detail.

2. Bowen-Wilmanski approach

Classically for the investigation of solids the Lagrangian description and for the analysis of fluids the Eulerian description is used (for a detailed presentation of Euler and Lagrange representations see K. HUTTER and K. JÖHNK [80]). Now a multi-component body consisting of a solid and one or more fluid components is considered. It is obvious that it would be disadvantageous to use different types of description for different components. Therefore, very often and especially for flow problems Eulerian description is used. However, also the consistent reference of the movement of the fluid phases to the reference configuration of the solid is possible. Such a Lagrangian description for porous media, e.g., has been introduced by K. WILMANSKI $[125,126]$.

The main difference between the classical mixture theory for fluid components (miscible components) and theories for porous media (immiscible fluid components) is the existence of additional fields characterizing the porous material. They describe the volume fraction of each component in a microscopic control volume (the representative elementary volume REV). For A different components, the volume fractions obviously have to satisfy the condition

$$
\sum_{\alpha=1}^{\mathrm{A}} n^{\alpha}=1, \quad 1 \leq \alpha \leq \mathrm{A},
$$


which often is called saturation condition and in which $n^{\alpha}$ denotes the volume fraction of component $\alpha$. As a consequence of the additional variables, the equations have to be extended by the volume fractions and in order to solve the problem with an increased number of variables either additional constitutive laws have to be introduced or additional differential equations (evolution or balance laws) have to be proposed. One example for the first solution has been introduced by R.M. BOWEN [36]. He assumes that the volume fractions are proportional to the respective partial mass densities

$$
\rho^{\alpha}=n^{\alpha} \rho^{\alpha R}, \quad \sum_{\alpha=1}^{\mathrm{A}} n^{\alpha}=1, \quad \rho^{\alpha R}=\text { const. }
$$

where $n^{\alpha}$ are the volume fractions of components $\alpha, \alpha=1, \ldots$, A and $\rho^{\alpha R}$ the constant real mass densities, i.e., the mass densities of the pure components. In [36], the components are assumed to be incompressible. In a further paper [37], compressible components are investigated and for each volume fraction an own evolution equation is introduced. This is the first attempt following the second solution. Another approach is the model with a balance equation for porosity introduced by K. WILMANSKI $[125,126]$. In this model, the Lagrangian description is used. Fields are defined on a reference configuration of the skeleton, i.e., the solid confinement for the motion of fluid components. One reason for the choice of this type of description is that large deformations of the solid which come along with geometric nonlinearities can be better described in this way. The use of the Langrangian description was already indicated in the book by O. CousSY [44] and then systematically introduced to porous models by K. WILMANSKI. In his model, $\dot{\mathbf{x}}^{S}$ is the velocity of the solid and $\mathbf{x}^{\alpha}$ is the velocity of a fluid point of component $\alpha$ with reference to the configuration of the solid. Therewith, the relative velocity of fluid component $\alpha$ and solid $S$ can be formulated by the so-called Lagrangian velocity field of the fluid component $\dot{\mathbf{X}}^{\alpha}$. Then, the balance equations have the following form $\underline{\text { solid }}$

$$
\begin{array}{ll}
\text { mass } & \frac{\partial \rho^{S}}{\partial t}=\hat{\rho}^{S}, \\
\text { momentum } & \frac{\partial}{\partial t}\left(\rho^{S} \dot{\mathbf{x}}^{S}\right)=\operatorname{Div} \mathbf{P}^{S}+\rho^{S} \mathbf{b}^{S}+\hat{\mathbf{p}}^{S}, \\
\text { energy } & \frac{\partial}{\partial t}\left[\rho^{S}\left(\frac{1}{2} x^{S 2}+\varepsilon^{S}\right)\right]+\operatorname{Div} \mathbf{Q}^{S}= \\
& =\operatorname{Div}\left(\mathbf{P}^{S T} \dot{\mathbf{x}}^{S}\right)+\rho^{S} \mathbf{b}^{S} \cdot \dot{\mathbf{x}}^{S}+\rho^{S} r^{S}+\hat{\varepsilon}^{S},
\end{array}
$$

$\underline{\text { fluids }}$

$$
\begin{array}{ll}
\text { mass } & \frac{\partial \rho^{\alpha}}{\partial t}+\operatorname{Div}\left(\rho^{\alpha} \dot{\mathbf{X}}^{\alpha}\right)=\hat{\rho}^{\alpha}, \\
\text { momentum } & \frac{\partial}{\partial t}\left(\rho^{\alpha} \dot{\mathbf{x}}^{\alpha}\right)+\operatorname{Div}\left(\rho^{\alpha} \hat{\mathbf{x}}^{\alpha} \otimes \dot{\mathbf{X}}^{\alpha}-\mathbf{P}^{\alpha}\right)=\rho^{\alpha} \mathbf{b}^{\alpha}+\hat{\mathbf{p}}^{\alpha}, \\
\text { energy } & \frac{\partial}{\partial t}\left[\rho^{\alpha}\left(\frac{1}{2} \dot{x}^{\alpha 2}+\varepsilon^{\alpha}\right)\right]+\operatorname{Div}\left[\rho^{\alpha}\left(\frac{1}{2} \dot{x}^{\alpha 2}+\varepsilon^{\alpha}\right) \dot{\mathbf{X}}^{\alpha}+\mathbf{Q}^{\alpha}\right]= \\
& =\operatorname{Div}\left(\mathbf{P}^{\alpha T} \hat{\mathbf{x}}^{\alpha}\right)+\rho^{\alpha} \mathbf{b}^{\alpha} \cdot \dot{\mathbf{x}}^{\alpha}+\rho^{\alpha} r^{\alpha}+\hat{\varepsilon}^{\alpha}, \\
\text { porosity } & \frac{\partial n}{\partial t}+\operatorname{Div}(\mathbf{J})=\hat{n} .
\end{array}
$$


Moreover, the following denotations have been used:

$\rho^{S} \quad$ - partial mass density of the solid per unit volume of the reference configuration ( $\mathrm{rc}$ )

$\hat{\rho}^{S} \quad$ - mass source of the solid per unit volume of rc

$\mathbf{P}^{S} \quad$ - partial Piola-Kirchhoff stress tensor of the solid

$\hat{\mathbf{p}}^{S} \quad$ - momentum source of the solid

$\mathbf{b}^{S} \quad$ - partial volume force of the solid per unit mass of the solid

$\varepsilon^{S} \quad$ - specific inner energy of the solid per unit mass of the solid

$\mathbf{Q}^{S} \quad$ - partial heat flux in the solid per unit surface of the solid

$\hat{\varepsilon}^{S} \quad$ - partial energy source of the solid per unit volume of rc

$r^{S} \quad$ - energy radiation in the solid per unit mass of the solid

$\rho^{\alpha} \quad$ - partial mass density of component $\alpha$ per unit volume of the reference configuration of the solid (rcs)

$\hat{\rho}^{\alpha} \quad$ - partial mass source of component $\alpha$ per unit mass of res

$\mathbf{P}^{\alpha} \quad$ - partial Piola-Kirchhoff stress tensor of component $\alpha$ in relation to rcs

$\hat{\mathbf{p}}^{\alpha} \quad$ - momentum source of component $\alpha$

$\mathbf{b}^{\alpha} \quad$ - partial volume force of component $\alpha$ per unit mass in rcs

$\varepsilon^{\alpha} \quad$ - specific inner energy of component $\alpha$ per unit mass in rcs

$\mathbf{Q}^{\alpha} \quad$ - partial heat flux of component $\alpha$ per unit surface of res

$\hat{\varepsilon}^{\alpha} \quad$ - partial energy source of component $\alpha$ per unit volume of res

$r^{\alpha} \quad$ - energy radiation of component $\alpha$ per unit mass of res.

Equation (16) is the porosity balance which has the same form as the other balance equations. For the porosity field $n$, it consists of a time change $\frac{\partial n}{\partial t}$, a flux $\mathbf{J}$ and a source $\hat{n}$. For further information on this model, see, e.g., [125] and for a motivation based on image analysis for the balance equation of porosity [127] (pages 201-207).

3. Continuum model in Eulerian description

Especially for the description of propagation of sound waves or other processes where only small deformations of the solid occur the continuous Eulerian description of the porous medium is advantageous, for example, in the theses [1,7], but also in the articles $[6,11,49]$ for different numbers of pore fluids models for porous media in Eulerian description are used. For a porous medium consisting of one solid $S$ and A fluid components $\alpha$, the respective velocities of these components are $\mathbf{v}^{S}$ and $\mathbf{v}^{\alpha}$. The balance equations have the following form

$$
\begin{array}{ll}
\begin{array}{l}
\text { solid } \\
\text { mass }
\end{array} & \frac{\partial \rho^{S}}{\partial t}+\operatorname{div} \rho^{S} \mathbf{v}^{S}=\hat{\rho}^{S}, \\
\text { momentum } & \frac{\partial}{\partial t}\left(\rho^{S} \mathbf{v}^{S}\right)+\operatorname{div}\left(\rho^{S} \mathbf{v}^{S} \otimes \mathbf{v}^{S}-\mathbf{T}^{S}\right)=\rho^{S} \mathbf{b}^{S}+\hat{\mathbf{p}}^{S}, \\
\text { energy } & \frac{\partial}{\partial t}\left[\rho^{S}\left(\frac{1}{2} v^{S 2}+\varepsilon^{S}\right)\right]+\operatorname{div}\left[\rho^{S}\left(\frac{1}{2} v^{S 2}+\varepsilon^{S}\right) \mathbf{v}^{S}+\mathbf{q}^{S}\right] \\
& =\operatorname{div}\left(\mathbf{T}^{S T} \mathbf{v}^{S}\right)+\rho^{S} \mathbf{b}^{S} \cdot \mathbf{v}^{S}+\rho^{S} r^{S}+\hat{\varepsilon}^{S},
\end{array}
$$

fluids

$$
\begin{array}{ll}
\text { mass } & \frac{\partial \rho^{\alpha}}{\partial t}+\operatorname{div} \rho^{\alpha} \mathbf{v}^{\alpha}=\hat{\rho}^{\alpha}, \\
\text { momentum } & \frac{\partial}{\partial t}\left(\rho^{\alpha} \mathbf{v}^{\alpha}\right)+\operatorname{div}\left(\rho^{\alpha} \mathbf{v}^{\alpha} \otimes \mathbf{v}^{\alpha}-\mathbf{T}^{\alpha}\right)=\rho^{\alpha} \mathbf{b}^{\alpha}+\hat{\mathbf{p}}^{\alpha}, \\
\text { energy } & \begin{array}{c}
\frac{\partial}{\partial t}\left[\rho^{\alpha}\left(\frac{1}{2} v^{\alpha 2}+\varepsilon^{\alpha}\right)\right]+\operatorname{div}\left[\rho^{\alpha}\left(\frac{1}{2} v^{\alpha 2}+\varepsilon^{\alpha}\right) \mathbf{v}^{\alpha}+\mathbf{q}^{\alpha}\right] \\
=\operatorname{div}\left(\mathbf{T}^{\alpha T} \mathbf{v}^{\alpha}\right)+\rho^{\alpha} \mathbf{b}^{\alpha} \cdot \mathbf{v}^{\alpha}+\rho^{\alpha} r^{\alpha}+\hat{\varepsilon}^{\alpha} .
\end{array}
\end{array}
$$


The following additional denotations have been used:

$\rho^{S} \quad$ - partial mass density of the solid per unit volume of the current configuration (cc)

$\hat{\rho}^{S} \quad$ - mass source of the solid per unit volume of $\mathrm{cc}$

$\mathbf{T}^{S} \quad$ - partial Cauchy stress tensor of the solid

$\hat{\mathbf{p}}^{S} \quad$ - momentum source of the solid

$\mathbf{b}^{S} \quad$ - partial volume force of the solid per unit mass of the solid

$\varepsilon^{S} \quad$ - specific inner energy of the solid per unit mass of the solid

$\mathbf{q}^{S} \quad$ - partial heat flux in the solid per unit surface of the solid

$\hat{\varepsilon}^{S}$ - partial energy source of the solid per unit volume of cc

$r^{S} \quad$ - energy radiation in the solid per unit mass of the solid

$\rho^{\alpha} \quad$ - partial mass density of component $\alpha$ per unit volume of the current configuration (cc)

$\hat{\rho}^{\alpha} \quad$ - mass source of component $\alpha$ per unit mass of cc

$\mathbf{T}^{\alpha} \quad$ - partial Cauchy stress tensor of component $\alpha$

$\hat{\mathbf{p}}^{\alpha} \quad$ - momentum source of component $\alpha$

$\mathbf{b}^{\alpha} \quad$ - partial volume force of component $\alpha$ per unit mass in cc

$\varepsilon^{\alpha} \quad$ - partial inner energy of component $\alpha$ per unit mass of cc

$\mathbf{q}^{\alpha} \quad$ - partial heat flux of component $\alpha$ per unit surface of cc

$\hat{\varepsilon}^{\alpha} \quad$ - partial energy source of component $\alpha$ per unit volume of cc

$r^{\alpha} \quad$ - energy radiation in component $\alpha$ per unit mass of cc.

\subsection{Empirical approaches}

1. Biot theory

The probably most frequently used model to describe processes in fluid-saturated porous materials is the Biot model. Since its introduction by MAURICE A. BIOT, many researchers adopted this model. In 1956, BIOT used the model the first time to describe the wave propagation in porous media, especially in soils. The Biot model is of intuitive or empirical nature. It is limited to specific multi-phase systems and insofar has a narrow range of validity. However, K. WILMANSKI could show that under certain conditions Biot's model can be derived from thermodynamic models and principles [128]. Here, at first the Biot model is presented in the same form as given in 1956 by BIOT [28]. One should mention that BIOT in his subsequent papers changed many times some denotations of material parameters.

Most interesting are the momentum balances. In the above-mentioned paper, they are given in the following form

$$
\begin{aligned}
N \nabla^{2} \mathbf{u}+\operatorname{grad}[(A+N) e+Q \varepsilon] & =\frac{\partial^{2}}{\partial t^{2}}\left(\rho_{11} \mathbf{u}+\rho_{12} \mathbf{U}\right)+b \frac{\partial}{\partial t}(\mathbf{u}-\mathbf{U}), \\
\operatorname{grad}[Q e+R \varepsilon] & =\frac{\partial^{2}}{\partial t^{2}}\left(\rho_{12} \mathbf{u}+\rho_{22} \mathbf{U}\right)-b \frac{\partial}{\partial t}(\mathbf{u}-\mathbf{U}) .
\end{aligned}
$$

In these equations, $\mathbf{u}$ and $\mathbf{U}$ are the displacements of the solid and the fluid, respectively. The volume changes in the solid and in the fluid, $e$ and $\varepsilon$, are related to the displacements by

$$
e=\operatorname{div} \mathbf{u}, \quad \varepsilon=\operatorname{div} \mathbf{U} .
$$

This means that the equations can be expressed also using only the displacements as variables

$$
\begin{aligned}
N \nabla^{2} \mathbf{u}+(A+N) & \nabla(\nabla \cdot \mathbf{u})+ \\
+Q \nabla(\nabla \cdot \mathbf{U}) & =\frac{\partial^{2}}{\partial t^{2}}\left(\rho_{11} \mathbf{u}+\rho_{12} \mathbf{U}\right)+b \frac{\partial}{\partial t}(\mathbf{u}-\mathbf{U}), \\
Q \nabla(\nabla \cdot \mathbf{u})+R \nabla(\nabla \cdot \mathbf{U}) & =\frac{\partial^{2}}{\partial t^{2}}\left(\rho_{12} \mathbf{u}+\rho_{22} \mathbf{U}\right)-b \frac{\partial}{\partial t}(\mathbf{u}-\mathbf{U}) .
\end{aligned}
$$


$A, N$ and $Q$ are material parameters. $\rho_{11}, \rho_{22}$ and $\rho_{12}$ are mass coefficients of which the first two are related to the solid and to the fluid, respectively. The coefficient $\rho_{12}$ is attributed to the tortuosity $a$. A typical simplified relation was proposed by BERRYMAN in 1980 [24] and has the form

$$
\rho_{12}=\rho^{F}(1-a), \quad a=\frac{1}{2}\left(\frac{1}{n_{0}}+1\right) .
$$

In continuum mechanics, another form of description of the same equations is useful and mostly used. It does not rely on the displacements of the components but (as in the models shown above) on their macroscopic (Eulerian) partial velocities $\mathbf{v}^{S}$ (of the solid) and $\mathbf{v}^{F}$ (of the fluid). In this version, the symmetric AlmansiHamel tensor of small deformations of the skeleton, $\mathbf{e}^{S}$, and the volume change of the fluid, $\varepsilon$, are the measures of deformation. The Almansi-Hamel tensor vanishes in the reference configuration, and its limit for small deformations is commonly used in the linear theory of elasticity.

In this description, the Biot model has the following form

$$
\begin{aligned}
& \frac{\partial \rho^{F}}{\partial t}+\rho_{0}^{F} \operatorname{div} \mathbf{v}^{F}=0, \quad\left|\frac{\rho^{F}-\rho_{0}^{F}}{\rho_{0}^{F}}\right| \ll 1, \quad\left\|\mathbf{e}^{S}\right\| \ll 1, \quad \varepsilon:=\frac{\rho_{0}^{F}-\rho^{F}}{\rho_{0}^{F}}, \\
& \rho_{0}^{S} \frac{\partial \mathbf{v}^{S}}{\partial t}=\lambda^{S} \operatorname{grad} \operatorname{tr} \mathbf{e}^{S}+2 \mu^{S} \operatorname{div} \mathbf{e}^{S}+Q \operatorname{grad} \varepsilon+ \\
& +\pi\left(\mathbf{v}^{F}-\mathbf{v}^{S}\right)-\rho_{12}\left(\frac{\partial \mathbf{v}^{F}}{\partial t}-\frac{\partial \mathbf{v}^{S}}{\partial t}\right) \\
& \rho_{0}^{F} \frac{\partial \mathbf{v}^{F}}{\partial t}=\kappa \rho_{0}^{F} \operatorname{grad} \varepsilon+Q \operatorname{grad} \operatorname{tr} \mathbf{e}^{S}-\pi\left(\mathbf{v}^{F}-\mathbf{v}^{S}\right)+\rho_{12}\left(\frac{\partial \mathbf{v}^{F}}{\partial t}-\frac{\partial \mathbf{v}^{S}}{\partial t}\right) .
\end{aligned}
$$

Here, $\rho^{F}$ is the partial mass density of the fluid and $\rho_{0}^{F}=n_{0} \rho_{0}^{F R}$ and $\rho_{0}^{S}=\left(1-n_{0}\right) \rho_{0}^{S R}$ are constant reference values of the partial mass densities where $\rho_{0}^{F R}, \rho_{0}^{S R}$ are the initial realistic (true) mass densities and $n_{0}$ is the initial porosity. The macroscopic compressibility is denoted by $\kappa$, and $\varepsilon$ describes the volume change of the fluid component. $\lambda^{S}$ and $\mu^{S}$ are macroscopic elastic constants of the skeleton. The deformations of the skeleton are assumed to be small.

The time derivatives of the deformations are connected to the velocities in the following way

$$
\frac{\partial \mathbf{e}^{S}}{\partial t}=\operatorname{sym} \operatorname{grad} \mathbf{v}^{S}=\frac{1}{2}\left[\operatorname{grad} \mathbf{v}^{S}+\left(\operatorname{grad} \mathbf{v}^{S}\right)^{T}\right], \quad \frac{\partial \varepsilon}{\partial t}=\operatorname{div} \mathbf{v}^{F} .
$$

The theory of Biot found many applications, especially for the description of processes in geomaterials, e.g., RICE and CleARY [105], PECKER and DERESIEWICZ [100] who also considered thermal effects or SCHREFLER [112], relied on this model. Biot's theory has been verified by PLONA in 1980 conducting experiments with sintered glass [24,101]. The main difference of the Biot model (27) and the model (17)-(22) is the occurrence of the relative accelerations and the static coupling parameter $Q$. A detailed comparison of the models connected to the propagation of sound waves can be found in [11] and in connection to surface waves in [5].

2. Reaction diffusion equations

The balance equations of the continuum models in Eulerian form shown above can be transformed under simplifying assumptions to a reaction diffusion equation. This type of equation is often used by mathematicians to describe processes in porous and granular media (e.g., ECK, GARCKE AND KNABNER [53]). Sometimes this method is actually put on the same level with the continuum mechanical description of porous media (e.g., SAHIMI [110] or Chapter 1 by LICHTNER in [88]). As the notation "reaction-diffusion equation" implies, such an equation can only be derived if the mass source in (20) is not zero, i.e., that an mass exchange between components occurs. Let us assume that we have an adsorption process (for details see, e.g., ALBERS [3]). It is assumed that a fluid-adsorbate mixture flows through the channels of a porous medium and that during the flow process adsorbate particles stick to the inner surface and change their kinematics from this of the fluid to that of the solid. The concentration $c$ of the adsorbate in the fluid is small. It is defined as

$$
c=\frac{\rho^{A}}{\rho^{L}}, \quad \rho^{L}:=\rho^{F}+\rho^{A},
$$


where $\rho^{A}, \rho^{F}$ and $\rho^{L}$ are the mass densities of the adsorbate, the fluid and the sum of both, respectively. Under restrictive assumptions a derivation of the reaction diffusion equation from the continuum equations is possible: as a first assumption the movement of the solid is neglected. In consequence, the equations for the solid are not needed. Thus, effects like the coupling of mass exchange and stresses in the solid are not covered any more. Moreover, the form of the Cauchy stress tensor is limited to the spherical pressure $\mathbf{T}^{F}=-p^{F} \mathbf{1}$. Indeed, this is the most used form of the stress in the fluid but for continuum models it is not the only possibility. If, additionally, the acceleration terms in the momentum balance of the fluid are neglected the Darcy law is obtained from the momentum balance for the fluid (e.g., from (21) if $\alpha$ is replaced by $F$ )

$$
\frac{\partial p^{F}}{\partial x}=-\pi v^{F}
$$

where $v^{F}$ is the seepage velocity, $\frac{\partial p^{F}}{\partial x}$ the pressure gradient and $\pi=\frac{\rho^{F R} g}{K}$ is the permeability or rather resistance parameter which depends on the true mass density $\rho^{F R}$ of the fluid, the earth acceleration $g$ and the hydraulic conductivity $K$. Originally this law was not derived from the momentum balance but Darcy found it empirically. Therefore, the momentum equations are not necessary to find the reaction diffusion equation.

The Darcy law is inserted into the mass balance (20) for the liquid component $L$. Simultaneously replacing the mass source $\hat{\rho}_{t}^{L}$ by a concentration source $\hat{c}=\frac{\hat{\rho}_{t}^{A}}{\rho^{L}}$ leads to

$$
\frac{\partial \rho^{L}}{\partial t}-\operatorname{div}\left(\rho^{L} \frac{1}{\pi} \operatorname{grad} p^{F}\right)=\rho^{L} \hat{c} .
$$

Considering the additional restriction that the overall mass density $\rho=\sum_{\alpha=1}^{\mathrm{A}} \rho^{\alpha}$ is constant and that $\rho^{L}=c^{L} \rho$ (which follows from the homogeneity of the system), an equation for the concentration can be written

$$
\frac{\partial c^{L}}{\partial t}=\operatorname{div}\left[D\left(c^{L}\right) \operatorname{grad} c^{L}\right]+c^{L} \hat{c} \text { with } D=c^{L} \frac{1}{\pi} \frac{\partial p^{F}}{\partial c^{L}} .
$$

The diffusion coefficient $D$ obviously depends on the concentration, the permeability of the material and the pressure change when a change of the concentration occurs. Equation (32) is the desired relation which, of course, due to the restrictions is limited in application. Several characteristic features of porous media cannot be described by this relation even if it is generalized by additional scalar equations.

Of course, there appear differences in the description of physical adsorption processes and chemical reactions: If physical processes in porous media are described, in general temperature-independent processes are investigated and it is not necessary to introduce a temperature. This is not the case if chemical reactions are considered. If phases react with each other, mostly this is connected with generation of heat. To describe such a process, an additional variable is necessary - the temperature - and this, in turn, leads to additional equations. While for the description of physical processes the above shown energy balances mostly are not used, for the description of chemical reactions they are necessary. As mentioned in WILMANSKI [130], the problem of thermodiffusion within macroscopic models is still very much open. The reason is that difficulties with an appropriate definition of the temperature on the macroscopic level of description occur. One open question is, for example, how to deal with the problem of different temperatures for different components (Fig. 2).

\section{Basics of chemical reactions used in mixture theories}

\subsection{Chemical potential for fluid mixtures}

One of the fundamental quantities which is needed to describe chemical reactions is the chemical potential of a material or component because it characterizes the possibilities of a substance to react with other substances, to undergo a phase change or to reallocate in space by diffusion. In the textbook by JOB and RUFFLER [83], the most important attributes of the chemical potential are summarized as follows: the tendency of a substance

- To decay or to transform together with other substances, 


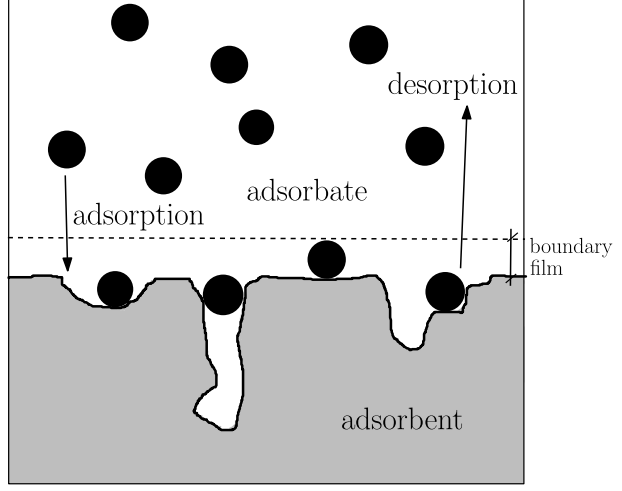

(a)

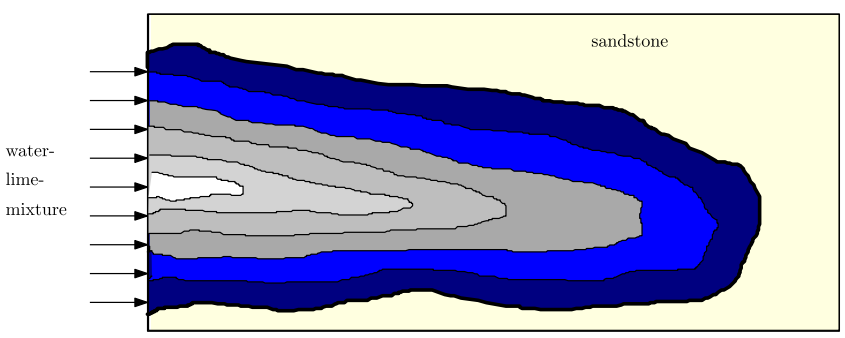

(b)

Fig. 2 a Illustration of a physical adsorption process: a fluid-adsorbate mixture flows through the channels of a porous medium. During the flow process adsorbate particles stick to the inner surface and change their kinematics from this of the fluid to that of the solid. According to the Langmuir theory the particles stick to the solid in one single layer. $\mathbf{b}$ Illustration of a chemical reaction process in a pore of a sandstone. It is obvious that the reaction at first takes place on the surface of the solid. If then more reactant is brought in more and more layers are built until finally the pore is completely filled. For fotos of this process, see Fig. 6 in [50]

- To convert into another status type,

- To reallocate in space

can be described by the same quantity, namely the chemical potential $\mu$. The intensity of this tendency, i.e., the value of $\mu$, is not invariable but is influenced either by the nature of the substance and by the environment but neither by the nature of its reaction partners nor by the evolving products. A transformation, conversion or reallocation voluntarily only can occur if the tendency in the initial situation is stronger than in the end situation.

Because of its importance and its difficulty to grasp several researchers undertook a thorough analysis of this quantity, e.g., COOK and DiCKERSON [43], BAIERLEIN [15], JOB and HERRMANN [82] or CHEN [41]. As indicated above, a reaction, phase change or diffusion can only take place voluntarily (without constraint) if the chemical potential in the initial state is larger than in the end state; i.e., a reaction occurs by itself if the sum of the chemical potentials of the initial situation is bigger than that of the end condition. This is comparable to another, better known, potential appearing in groundwater flows: the hydraulic or piezometric head. Its distribution through an aquifer determines where groundwater will flow. If the hydraulic head is constant, there is no flow. However, if there is a difference in hydraulic head, the water will flow from the higher to the lower potential and the difference in head and the length of the flow path defines the hydraulic gradient. Coming back to the chemical potential we consider, as is done in [43], a system which can exchange energy and particles with a reservoir and whose volume can change. Through heating the system gains energy, i.e., its internal energy $U$ increases by $T \mathrm{~d} S$, where $T$ is the temperature of the system and $S$ its entropy. If the system expands by volume $d V$ at pressure $p$, the work done by the system is $p \mathrm{~d} V$. If the system gains $\mathrm{d} N$ particles (all of one type), then $U$ increases by $\mu \mathrm{d} N$, where $\mu$ is the chemical potential. The change in the internal energy is then

$$
\mathrm{d} U=T \mathrm{~d} S-p \mathrm{~d} V+\mu \mathrm{d} N .
$$

This relation is commonly called the thermodynamic identity, valid for infinitesimal, reversible processes. For the chemical potential, it follows

$$
\mu=\left(\frac{\mathrm{d} U}{\mathrm{~d} N}\right)_{S, V} .
$$

Thus, it is calculated as the change in internal energy of the system, when one more particle is added, while holding the volume and the entropy constant. To clarify the expression "entropy" the Merriam-Webster dictionary is adducted. Accordingly, in thermodynamics entropy is defined as "a measure of the unavailable energy in a closed thermodynamic system that is also usually considered to be a measure of the system's disorder, that is a property of the system's state, and that varies directly with any reversible change in heat in the system and inversely with the temperature of the system." Since it is hard to control the volume and entropy to be constant while particles are added often another form of the chemical potential is used. As 


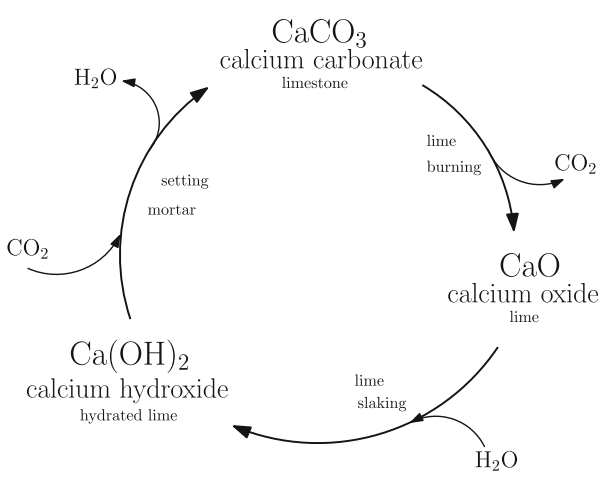

Fig. 3 Simplified lime cycle

pointed out in [15] one of the characterizations of the chemical potential is when a reaction occurs that it measures the contribution to the rate of change of the function whose extremum determines equilibrium. In different circumstances, different thermodynamic functions attain extrema. For example, if a reaction comes to equilibrium at fixed temperature and pressure, the Gibbs free energy $G=U+p V-T S$ attains a minimum and the chemical potential can be written as

$$
\mu=\left(\frac{\mathrm{d} G}{\mathrm{~d} N}\right)_{T, p} .
$$

If equilibrium is gained at fixed temperature and volume, then the Helmholtz free energy $F=U-T S$ has an extremum and the chemical potential reads

$$
\mu=\left(\frac{\mathrm{d} F}{\mathrm{~d} N}\right)_{T, V} .
$$

Different forms of the chemical potential (34), (35) and (36) are equivalent and may be useful in different physical situations. For the meaning and conversion of the different types of energy with different independent variables see SCHROEDER [113].

The chemical reaction which takes place in the example mentioned in the introduction, namely that the pores of a weathered monument are filled by a water-lime-mixture is considered here. As described above, the mixture penetrates into the near surface pore structure of the stone and the calcium hydroxide reacts with carbon dioxide and builds calcium carbonate and water and the following chemical reaction occurs

$$
\mathrm{Ca}(\mathrm{OH})_{2}+\mathrm{CO}_{2} \rightarrow \mathrm{CaCO}_{3}+\mathrm{H}_{2} \mathrm{O} .
$$

This is one branch of the lime cycle shown in Fig. 3. When limestone (calcium carbonate) is burnt, it turns into quicklime (calcium oxide) and releases carbon dioxide. If water is added to the burnt lime, hydrated lime (calcium hydroxide) is produced. The process which is of interest here is the building of a firm layer which happens if the calcium hydroxide reacts with carbon dioxide and builds calcium carbonate and water. This turns the lime back to lime stone.

The following values of chemical potentials under normal conditions can be obtained from chemical tables (e.g.,job-stiftung.de/index.php?datensammlung):

$$
\begin{array}{ll}
\mu_{\mathrm{Ca}(\mathrm{OH})_{2}} & =-897 \mathrm{kG}=\frac{\mathrm{kJ}}{\mathrm{mol}} \\
\mu_{\mathrm{CO}_{2}} & =-394 \mathrm{kG} \\
\mu_{\mathrm{CaCO}_{3}} & =-1129 \mathrm{kG} \\
\mu_{\mathrm{H}_{2} \mathrm{O}} & =-237 \mathrm{kG} .
\end{array}
$$

The unit G stands for "Gibbs" and is identical to $\mathrm{J} / \mathrm{mol}$. In order to decide whether the reaction proceeds from left to right or from right to left, we have to compare the total potential $\mu_{R}$ of the reactants with the total potential $\mu_{P}$ of the products. If

- $\mu_{R}>\mu_{P}$, then transformation of substance $R$ into substance $P$ or transport from place $R$ to place $P$ takes place

- $\mu_{R}=\mu_{P}$, then no transformation, no transport takes place, the system is in chemical equilibrium 
- $\mu_{R}<\mu_{P}$, transformation of substance $P$ into substance $R$ or transport from place $P$ to place $R$.

$R$ and $P$ can be either pure substances or a combination of substances: a mixture, an alloy, a solution or even a set of substances in various distinct environments [82]. For the chemical reaction (37) results

$$
\begin{aligned}
& \mu_{R}=\mu_{\mathrm{Ca}(\mathrm{OH})_{2}}+\mu_{\mathrm{CO}_{2}}=-1291 \mathrm{kG} \\
& \mu_{P}=\mu_{\mathrm{CaCO}_{3}}+\mu_{\mathrm{H}_{2} \mathrm{O}}=-1366 \mathrm{kG}
\end{aligned}
$$

Thus, we have $\mu_{R}>\mu_{P}$. This chemical reaction takes place voluntarily because the tendency for a change is more pronounced in the initial state than in the final state, i.e., the chemical potential in the initial state $R$ is greater than in the final state $P$. This result was to be expected since the reaction equation describes the setting of mortar which we know proceeds spontaneously (for more examples see: [82]).

In the notation used above for the mixture theory Eqs. (2)-(4) the chemical potential $\mu_{i}$ of a system component $i$ is generally defined by the GIBBS fundamental thermodynamic relation of the inner energy $\varepsilon$

$$
\mathrm{d} \varepsilon=T \mathrm{~d} \eta-p \mathrm{~d} V+\sum \mu_{i} \mathrm{~d} n_{i}
$$

where $T$ denotes the absolute temperature, $\eta$ is the entropy, $p$ the pressure, $V$ the volume and $n_{i}$ the amounts of the chemical components $i$. This is the same equation as (33) only in different notation. $\varepsilon, \eta$ and $p$ are state functions which only depend on the current equilibrium state of the system, here they depend only on $\rho$ and $T$. In thermodynamic equilibrium there are no net macroscopic flows of matter or of energy, either within a system or between systems.

\subsection{Chemical potential for porous media}

\subsubsection{Chemical potential according to Bowen}

In [36] and [37], R.M. BowEN introduces and discusses porous media models for incompressible and compressible components by use of thermodynamics of mixtures. In the theory of mixtures each constituent is assigned a property called its chemical potential. Bowen shows how these quantities determine the stress tensor for the porous material and the pore pressure for each pore fluid. It was pointed out above that material equations must satisfy the second law of thermodynamics, i.e., the entropy principle. It was said that the entropy is a directed quantity which does not fulfill a conservation law but an inequality. For a porous medium consisting of one solid (in Bowen's papers denoted by index 1) and A fluid components (indices 2 to $1+\mathrm{A}=N$ ) it has the form (whether incompressible or compressible) [36]

$$
\rho \dot{\eta}+\operatorname{div} \sum_{\alpha=1}^{N}\left(\frac{\mathbf{q}^{\alpha}}{\theta^{\alpha}}+\rho^{\alpha} \theta^{\alpha} \mathbf{u}^{\alpha}\right)-\sum_{\alpha=1}^{N} \rho^{\alpha} \frac{r^{\alpha}}{\theta^{\alpha}} \geq 0,
$$

where $\rho=\sum_{\alpha=1}^{1+\mathrm{A}} \rho^{\alpha}$ is the density of the whole body, $\theta^{\alpha}$ is a positive number representing the temperature of the $\alpha$ th constituent, $\mathbf{u}^{\alpha}$ is the diffusion velocity which is defined by $\mathbf{u}^{\alpha}:=\mathbf{v}-\mathbf{v}^{\alpha}$ (compare (5) $)$ ). The use of this diffusion velocity differs from most other models for porous media. In most models a relative velocity between the solid component and fluid components is defined (see, e.g., DETMANN [49] or RAJAGOPAl and TAO [104]). The partial heat flux of component $\alpha$ and the energy radiation in component $\alpha$ are, again, denoted by $\mathbf{q}^{\alpha}$ and $r^{\alpha}$. If $\eta^{\alpha}$ denotes the entropy density of the constituent $\alpha$, then

$$
\eta(\mathbf{x}, t)=\frac{1}{\rho} \sum_{\alpha=1}^{N} \rho^{\alpha} \eta^{\alpha}(\mathbf{x}, t),
$$

defines the entropy density of the mixture. Both in [36] and [37] Bowen considers the special case where every constituent is constrained to have the same temperature as every other constituent, so that (39) takes the form

$$
\rho \dot{\eta}+\operatorname{div}\left(\frac{1}{\theta}\left(\mathbf{k}-\sum_{\alpha=1}^{N} \rho^{\alpha} \psi^{\alpha} \mathbf{u}^{\alpha}\right)\right)-\rho \frac{r}{\theta} \geq 0,
$$


where $\theta(\mathbf{x}, t)$ is the temperature of the mixture and $\psi^{\alpha}:=\varepsilon^{\alpha}-\theta \eta^{\alpha}$ is the partial Helmholtz free energy of component $\alpha$ (compare with (33), (36) and (38)) and $\mathbf{k}=\sum_{\alpha=1}^{N}\left(\mathbf{q}^{\alpha}+\rho^{\alpha} \varepsilon^{\alpha} \mathbf{u}^{\alpha}\right)$. Now Bowen eliminates the factor $\rho r$ (for details see [37]) and gets

$$
-\sum_{\alpha=1}^{N} \Psi^{\alpha}-\rho \eta \dot{\theta}-\operatorname{tr} \sum_{\alpha=1}^{N} \rho^{\alpha} \mathbf{K}^{\alpha} \mathbf{L}^{\alpha}-\mathbf{h} \cdot \mathbf{g} / \theta-\sum_{\alpha=1}^{N} \mathbf{u}^{\alpha} \cdot \hat{\mathbf{p}}^{\alpha} \geq 0 .
$$

Here, $\Psi^{\alpha}:=\rho^{\alpha} \psi^{\alpha}$ is the free energy of constituent $\alpha$ per unit volume, $\mathbf{g}=\operatorname{grad} \theta$ is the spatial temperature gradient and $\mathbf{h}=\mathbf{k}-\sum_{\alpha=1}^{N} \Psi^{\alpha} \mathbf{u}^{\alpha}$. The velocity gradient of component $\alpha$ at $(\mathbf{x}, t)$ is denoted by $\mathbf{L}^{\alpha}$. Most important for the present work is the occurrence of the chemical potential tensor for the $\alpha$ th constituent $\mathbf{K}^{\alpha}$ defined by

$$
\mathbf{K}^{\alpha}=\psi^{\alpha} \mathbf{1}-\frac{\mathbf{T}^{\alpha T}}{\rho^{\alpha}} .
$$

This form of the chemical potential for mixtures is not really remindful of the relation for fluid mixtures (34), (35) or (36). Unfortunately, Bowen does not go into detail about its derivation. However, in [36] he mentions that it is customary in many porous media applications to eliminate the chemical potential of the solid in favor of the stress tensor of the mixture in all field equations. And the chemical potentials for the fluids could be eliminated in favor of parameters called the pore pressures. With the definition of the pore pressure for the $\alpha$ th constituent

$$
P^{\alpha}=\lambda+\frac{\partial \Psi^{I}}{\partial \phi^{\alpha}}
$$

where $\Psi^{I}=\sum_{\alpha=1}^{N} \Psi^{\alpha}$ is the inner part of the free energy of the mixture, $\phi^{\alpha}$ is the volume fraction of component $\alpha$ and $\lambda$ is an indeterminant hydrostatic pressure acting on the mixture follows for the chemical potential of constituent $\alpha$

and for the solid

$$
\rho^{\alpha} \mathbf{K}^{\alpha}=\phi^{\alpha} P^{\alpha} \mathbf{1}, \quad \alpha=2 \ldots N,
$$

$$
\rho^{S} \mathbf{K}^{S}=\phi^{S} \lambda \mathbf{1}-2 \mathbf{F}^{S} \frac{\partial \Psi^{I}}{\partial \mathbf{C}^{S}} \mathbf{F}^{S T},
$$

where $\mathbf{F}^{S}$ is the deformation gradient and $\mathbf{C}^{S}$ the right Cauchy-Green deformation tensor (for information on measures of deformation see [12]).

\subsubsection{Description of chemical reactions appearing in mixture theory and exploitation of the second law of thermodynamics}

For mixtures of chemically reacting materials interchange of mass between the constituents of the mixtures occurs and, consequently, in the balance equations the transfer of mass between the constituents must be allowed. One detailed discussion on chemically reacting continua at different temperatures of the constituents and at a common temperature is given by ATKIN and CRAINE in $[13,14]$. Therein, the approaches of ERINGEN and INGRAM [55,81] and MULLER [91] are in the front but also other researchers who were pioneers in dealing with mixtures were discussed, e.g., GREEN and NAGHDI [66] and BOWEN [32]. Most of these researchers worked thereby on an extension of the rational approach to the thermodynamics of a single continuum by COLEMAN and NOLL [42] to the thermodynamics of mixtures. Coleman and Noll proposed a procedure which combines the balance equations in an intelligent way in order to draw, together with some general principles of the theory of materials, conclusions with respect to the structure of the constitutive equations and restrictions on them [79]. The Coleman-Noll procedure got standard for the exploitation of the second law of thermodynamics for simple materials. However, it is not the only one, another method was introduced by I.-S. LIU in 1972 [89]. A comparison of the two methods is given in [118]. MULLER [92] showed that the Coleman-Noll procedure in contrast to the Liu procedure cannot be used offhand for mixtures. Namely, he showed that the theory of Coleman and Noll does not permit a finite velocity of heat propagation in a viscous heat conducting fluid. The works [52] and [51] also report on problems for the application of the Coleman-Noll theory to mixtures. 
In spite of the known objections, e.g., KENYON [84] used the Coleman-Noll procedure also for mixtures and nevertheless got realistic results. Other researcher maybe were not aware of the objections and also used the Coleman-Noll theory (e.g., [70] or RICKEN ET AL. [106] in a paper on concentration driven phase transitions with application to methane oxidation in landfill cover layers).

\subsubsection{Discussion on the macro-scale chemical potential in porous media}

In Subsection 3.2.1, the definition of the chemical potential according to BOWEN has been introduced. This approach has been paid special attention because it was probably the first approach and all following formulations of the chemical potential for mixtures refer to this approach. However, it is by no means clear that this is the correct formulation. In BENNETHUM et al. [23], this question is discussed and, firstly, it is pointed out that the classical Gibbsian chemical potential, as introduced in Subsection 3.1, has the following characteristics: (1) it is a scalar and measures the energy required to insert a particle into the system; (2) its gradient is the driving force for diffusive flow according to Fick's law (see, e.g., CALLEN [40], DE GROOT and MAZUR [47] or MULLLER [93]) and (3) it is constant for a single constituent coexisting in two phases at equilibrium. Then, it is mentioned that in applying mixture theory to a two phase system, results are only obtained for a relative chemical potential, i.e.,

$$
\widetilde{\mu}_{j}^{\beta}=\frac{\partial A^{\beta}}{\partial c_{j}^{\beta}}=\mu_{j}^{\beta}-\mu_{N}^{\beta}, \quad j=1 \ldots N-1, \quad \beta=F, S .
$$

Here, $A^{\beta}$ is the intensive Helmholtz potential of phase $\beta$ and $\mu_{j}^{\beta}$ and $c_{j}^{\beta}$ denote the chemical potential and the mass concentration of the $j$ th constituent in the $\beta$ phase, respectively. It is pointed out that the primary reason for obtaining results in terms of the relative chemical potential is the interdependence of the concentrations $\sum_{j=1}^{N} c_{j}^{\beta}=1$. Now, it is shown that some of the results involving the relative chemical potential can be sharpened by choosing $\mu_{N}^{\beta}$ appropriately and subsequently deriving results for the absolute (nonrelative) chemical potentials. In this way, BOWEN [35] and BOWEN and WIESE [31] proposed as shown above the tensorial definition for the chemical potential which has been widely been used in many papers (here in a slightly different form than above)

$$
\mu_{j}^{\beta}=A_{j}^{\beta} \mathbf{1}-\frac{1}{\rho_{j}^{\beta}} \mathbf{T}_{j}^{\beta},
$$

where $\rho_{j}^{\beta}, A_{j}^{\beta}$ and $\mathbf{T}_{j}^{\beta}$ denote the volume-averaged density, Helmholtz free energy, and stress tensor of the $j$ th component in the $\beta$-phase, respectively. It can be shown that for a perfect fluid the chemical potential reduces to the classical thermostatics concept of Gibbs energy. However, apart from that the tensorial chemical potential does not satisfy the three characteristics of the classical chemical potential mentioned above. Obviously, approaches for chemical potentials of mixtures do not satisfy these characteristics because also for the approach shown in Subsection 3.2.1 this is not the case. It should be noted that equation (47) is also known as diffusional chemical potential which is relevant to applications in material science (see BALUFFI ET AL. [16]). Moreover, a discussion under which circumstances the tensorial chemical potential (48) follows from the Gibbs-Duhem equation can be found in the report [85] by O. KRISTENSSON.

In 1980, NUNZIATO and WALSH [97] presented also a theory for ideal multiphase mixtures with chemical reactions and diffusion. It relies on and extends the theory of multiphase mixtures by PASSMAN [98] who in turn used some of the kinematical concepts proposed by GOODMAN and COWIN [59]. Each constituent of a reacting mixture is expected to have its own temperature and, consequently, also energy exchange among the constituents is permitted. The authors discuss the approach for the chemical potential proposed by Bowen (48): They point out that this approach views the chemical potential as reflecting any change in the free energy due to the deformation of a constituent and not due just to changes in mass. They are of the opinion that this definition is appropriate for diffusing mixtures but not if chemical reactions occur. They bring the example that in the absence of diffusion, (48) bears no relation to the chemical potential appropriate for a chemically reacting solid mixture. In view of this, they have chosen an alternative approach and to adhere more closely to the spirit of the definition given in classical thermochemistry. That is, the chemical potential is a measure 
of how the free energy of the mixture changes as a result of changes in the mass concentrations. Thus, they adopted

$$
\mu^{\alpha}=\frac{\partial\left(\rho \psi^{I}\right)}{\partial \rho^{\alpha}}=\frac{\partial\left(\rho \psi^{\alpha}\right)}{\partial \rho^{\alpha}},
$$

as definition of the chemical potential which with the definition of the mass concentration

$$
c^{\alpha}=\frac{\rho^{\alpha}}{\rho^{R}}\left|\operatorname{det} \mathbf{F}^{\alpha}\right|,
$$

where $\rho^{R}$ is the reference density of the mixture and $\mathbf{F}^{\alpha}$ the deformation gradient of constituent $\alpha$, resulted in the formula

$$
\mu^{\alpha}=\frac{\partial\left(c \psi^{\alpha}\right)}{\partial c^{\alpha}} .
$$

This equation very much reminds (47). However, no relative chemical potential is formulated and the question arises whether this simple formulation correctly describes the interaction of the constituents in mixtures.

In BENNETHUM and CUSHMAN [22], a multiscale mixture theory including interfacial effects for swelling systems is introduced. Also for this problem the chemical potential is needed. The authors introduce it as follows: The chemical potentials of a single constituent in two different phases (such as water and ice) are equal at equilibrium. The flow of a constituent is essentially governed by the gradient of the chemical potential, and the flow of a bulk phase is governed essentially by the gradient of the Gibbs free energy. Thus, in an interactive system, the chemical potential must be defined in terms of the entire system. Of course, if there is very little interaction between phases/interfaces these generalized definitions reduce to their classical definition for a single phase system. In the present formulation, it is assumed that the energy of one phase/interface may be influenced by the composition of another phase/interface. Therefore a generalized chemical potential is defined using the following notation: Superscript and subscript Greek letters indicate the average of the microscopic intraparticle-phase at the mesoscale. The superscript or subscript $A$ always refers to the average of the mesoscopic particle property at the macro-scale. Other capital letters (e.g., $B, C$ ) refer to phases other than the particles at the mesoscale (e.g., liquid, air) and interfaces are indicated by using two sub/superscripts.

The chemical potential of the vicinal fluid is given by

$$
\begin{aligned}
\tilde{\mu}^{l A_{j}} & =\frac{\partial A^{l A}\left(c^{l A_{1}}, c^{l A_{2}}, \ldots, c^{l A_{N-1}}\right)}{\partial c^{l A_{j}}}+\frac{\rho^{l s A}}{\rho^{l A}} \frac{\partial A^{l s A}\left(c^{l A_{1}}, c^{l A_{2}}, \ldots, c^{l A_{N-1}}\right)}{\partial c^{l A_{j}}} \\
& =\mu^{l A_{j}}-\mu^{l A_{N}}, \quad j=1, \ldots N-1, \\
\mu^{l A_{N}} & =\frac{\partial A^{l A}\left(c^{l A_{1}}, c^{l A_{2}}, \ldots, c^{l A_{N}}\right)}{\partial c^{l A_{N}}}+\frac{\rho^{l s A}}{\rho^{l A}} \frac{\partial A^{l s A}\left(c^{l A_{1}}, c^{l A_{2}}, \ldots, c^{l A_{N}}\right)}{\partial c^{l A_{N}}}
\end{aligned}
$$

Similarly,

$$
\begin{aligned}
& \tilde{\mu}^{s A_{j}}=\frac{\partial A^{s A}}{\partial c^{s A_{j}}}+\frac{\rho^{l s A}}{\rho^{s A}} \frac{\partial A^{l s A}}{\partial c^{s A_{j}}}=\mu^{s A_{j}}-\mu^{s A_{N}}, \\
& \tilde{\mu}^{l s A_{j}}=\frac{\partial A^{l s A}}{\partial c^{l s A_{j}}}+\frac{\rho^{l A}}{\rho^{l s A}} \frac{\partial A^{l A}}{\partial c^{l s A_{j}}}+\frac{\rho^{s A}}{\rho^{l s A}} \frac{\partial A^{s A}}{\partial c^{l s A_{j}}}=\mu^{l s A_{j}}-\mu^{l s A_{N}}, \\
& \mu^{K_{j}}=\frac{\partial A^{K}}{\partial c^{K_{j}}}=\mu^{K_{j}}-\mu^{K_{N}}, \quad K=B, C, l A B, l A C, s A B, s A C, B C, \\
& \quad \text { for } j=1, \ldots N-1 .
\end{aligned}
$$

Thus, the chemical potential represents the change of energy of the entire medium with respect to the concentration of a species within a particular phase. This gives the expected form of Fick's law stating that flow of a species is governed by the gradient of the chemical potential.

Summarizing this Section one can realize that several formulations for the chemical potential in porous media have been proposed. However, it does not get clear whether they are all correct and whether they yield correct results. 


\section{Further examples of works including chemical reactions in porous media}

In a series of papers HASSANIZADEH and GRAY [60,68-70] (extended in 1990 by [71]) describe the modeling of porous media by use of the averaging technique. Interestingly, they discern three different types of models: (1) descriptive models of empirical nature, (2) continuum theories of mixtures and (3) the averaging technique. However, in my opinion, the use of the averaging theory is only one possibility to extend mixture theory in order to describe porous media. Only the combination of (2) and (3) enables the description of porous media. Another possibility of extension, often used by mathematicians, would be the use of homogenization techniques (see, e.g., [76]). However, in most theories of porous materials macroscopic models are related to microscopic relations by means of volume averaging (see, e.g., [129] for two-component media or [8] for three-component media). In (9), e.g., is shown, in which way the macroscopic mass densities are obtained from their microscopic, measurable counterparts, the true mass densities, by consideration of their portion of the volume. In Bowen's works macro-scale forms of the field equations are postulated. In the fourth paper, mentioned before, Gray also considers phase changes. Admittedly, nine restrictive assumptions are made which limit the applicability strongly.

In two further papers [60,61], GRAY introduced another concept in order to incorporate microscopic effects into macroscopic models. He explains that traditionally, porosity and fluid saturations, concepts that do not exist at the micro-scale, are included in macro-scale porous media theories to account for the presence of multiple phases at a point in a macro-scale continuum. However, he points out that these additional variables sometimes are insufficient to account for all important micro-scale processes that influence macro-scale behavior. Because of the dynamic motion of the fluids, many configurations and distributions of the fluids are possible for a given saturation. This point has been investigated also in ALBERS [9], [10] where the well known effect that the capillary pressure curve of partially saturated soils exhibits a hysteresis has been accounted for. For the same degree of saturation it has different values depending on the initial state of the soil, thus for drying of a wet soil or wetting of a dry soil. The influence of these different values of the capillary pressure on the propagation of sound waves has been studied by use of a linear hyperbolic model. Even if the model does not contain a hysteresis operator, the effect of hysteresis in the capillary pressure curve is accounted for. Gray pursues another approach and includes interfacial effects. In addition to porosity and saturation, specific interfacial area, the amount of interfacial area between two phases per unit volume of the system, is introduced as a macro-scale independent variable and this variable was used in some studies of mass transfer among phases of a porous medium (e.g., [21,22] or [63]). Another approach on macroscopic multi-phase flow with diffusion and chemical reactions not based on the mixture theory after Truesdell but incorporating moving interfaces is by C. M. MARLE [90]. He starts from the equations valid at the pore level and takes their mean values around each point to obtain macroscopic equations.

In the PhD thesis by F. BRUNNER [39], a mathematical model including three phases (gas, liquid and solid) consisting of multiple components, which may participate in chemical reactions (equilibrium or kinetic reactions, homogeneous or heterogeneous) is considered. Moreover, the transfer of mass across the phases is taken into account. The reduction scheme of KRÄUTLE, KNABNER and HOFFMANN [75] is applied to this model, which means that linear combinations of equations are taken to eliminate the equilibrium reaction rates and that a linear variable transformation of the original variables is performed. The timescales at which chemical reactions take place typically vary over several orders of magnitude. Therefore, besides kinetically controlled reactions, also equilibrium reactions are considered in the work of BRUNNER which are running so fast that a state of local equilibrium can be assumed.

One example dealing with a similar topic as the initially mentioned conservation of buildings by carbonization is presented by SAETTA, SChrefLER and VitALIANI in $[108,109]$ and CREAZZA ET AL. [45]. The governing equations of moisture, heat and carbon dioxide flows through concrete within the framework of a distributed parameter model are described. A numerical procedure based on the finite element method (for basics see, e.g., [111]) is developed to solve the set of equations and to investigate the influence of relative humidity and temperature. The amount of calcium carbonate that forms in a unit of time depends on the degree of carbonation, i.e., the availability of calcium hydroxide, the temperature, the carbon dioxide concentration and the relative humidity in the pore structure of the concrete. Another model of hygro-thermal behavior of concrete at high temperature with thermo-chemical and mechanical material degradation is introduced in [58] by Gawin, PESAVENTO and SCHREFLER. According to BAŽANT (e.g., [18] or newer publications $[17,19]$ ), the formulation of drying coupled with heat transfer is developed in terms of relative humidity rather than water content and the diffusivities of water and carbon dioxide are assumed to be strongly dependent on pore humidity, temperature and also on the degree of hydration of concrete. The authors realized that the use of 
evaporable water content as basic variable involved certain errors since Fick's law in terms of the evaporable water content could not be applied to obtain the differential equation governing the drying phenomenon when hydration proceeds. The authors state as reason that the porosity becomes non-uniform in time and equal values of evaporable water content do not correspond to equal value of pore humidity in the same points. Another work in which mass transport accompanied by chemical reactions in porous media is studied according to Fick's law and the so-called dusty-gas model is by Veldsink et al. [122]. A further work based on mixture theory and dealing with chemical reactions and diffusion in concrete is by A. J. VROMANS et al. [123]. The model describing the corrosion of concrete with sulfuric acid is applied to the chemical reactions which transforms slaked lime and sulfuric acid into gypsum releasing water. A similar topic is dealt with in VAN BALEN and VAN GEMERT [121] in which is shown how the carbonation process in lime mortar is influenced by the diffusion of carbon dioxide into the mortar pore system, by the kinetics of the lime carbonation reaction and by the drying and wetting process in the mortar. Another report on experimental results of $\mathrm{CaCO}_{3}$ precipitation kinetics can be found in [107] by RoQUEs and GIROu. Similar studies by HOUST and WITTMAN are on the influence of porosity and water content on the diffusivity of $\mathrm{CO}_{2}$ and $\mathrm{O}_{2}$ through hydrated cement paste [77] and on depth profiles of carbonates formed during natural carbonation [78]. The article [48] by DESPOTOU ET AL. deals with reaction mechanisms in mortars and the diffusion and reaction speeds during the carbonation process. An also rather experimental investigation of the physicochemical characteristics of ancient mortars with comparison to a reaction-diffusion model by ZOURIDAKIS AT AL. appeared in [132]. A slightly different reaction involving also sulfur is studied in [30] by BÖHM ET AL. where the corrosion in a sewer pipe is modeled as a moving-boundary system. By the same authors also appeared a strategy for predicting the penetration of carbonation reaction fronts in concrete [94].

A couple of references deal distinctively with geochemical reactions. One of them by GATICA et al. [57] is of mathematical nature and contains nonlinear reaction terms (see 2. in Subsection 2.2). It studies the interaction between chemical reaction and natural convection in porous media. A global driving force for natural convection are thermal or concentration gradients applied to the boundaries of the system. As changes in the density lead to natural convection, chemical reactions can provide a driving force for secondary flows. The critical value for the onset of instabilities is found by performing a linear stability analysis. A similar stability analysis has been performed by ALBERS [4] showing the stability behavior of flows within the abovementioned adsorption/diffusion model for porous materials [1,3]. A comprehensive book on geochemical reaction modeling is by C. M. BETHKE [25]. He presents a variety of model types of which each imposes on the system some variant of equilibrium but differs from others in the manner in which mass and heat transfer are specified. He mentions closed-system models in which no mass transfer occurs and titration models where one or more reactants are gradually added to the equilibrium system, as might occur as the grains in a rock gradually react with a pore fluid. Further types are fugacity models which are applied to geochemical processes in which a fluid remains in contact with a gaseous phase or kinetic reaction models. These are such in which the rates at which minerals dissolve into or precipitate from the equilibrium system are set by kinetic rate laws. Moreover, local equilibrium models, as for example a flow-through reaction path, are mentioned. In such a path, the model isolates from the system minerals that form over the course of the calculation, preventing them from reacting further. The last type of models are continuum models. BETHKE describes them as a natural marriage of local equilibrium and kinetic models used to describe the mass transport in hydrology and various fields of engineering. As an example of a model which predicts the distribution in space and time of the chemical reactions that occur along a groundwater flow path he mentions the book by J. BEAR [20]. For formulations either for systems involving dissolution and precipitation of minerals or those accounting for surface adsorption reactions he refers to publications of P.C. LICHTNER [87], [88]. Lichtner's works point to a model based on the reaction path approach developed for geochemical systems by H.C. HELGESON [72,73]. In this method an initial assemblage of minerals reacts irreversibly with an aqueous solution. A further work of HELGESON together with W.M. MURPHY [74] directly focuses on the influence of the surface area in geochemical processes. The dependence of adsorption-diffusion processes on this parameter and on bulk and surface permeabilities has been investigated also by ALBERS in [2]. LICHTNER [87] points out that the rates of reversible heterogeneous reactions are independent of the surface area. He differs between reactions which can be considered locally in equilibrium and are reversible and others which require kinetic description and are irreversible. Setting up his equations he also follows J. BEAR [20]. The model of LICHTNER doubtlessly makes acceptance of several restrictive adoptions (e.g., the immobility of the solid, the restriction to Darcy's law etc.), but it is one of the few models which is complete and also verified to really describe simultaneous chemical reactions and mass transport in hydrothermal systems. Thus, another reference to this model is quoted [86] and also data of a work by C.I. STEEFEL et al. which shows the outcome of the model [116]. In [103] P.A.C. RAATS reflects on 
applications of the theory of mixtures in soil physics. He points out that each of the phases of a porous medium is itself a mixture. The solid phase is a mixture of numerous inorganic and organic constituents, many of which are positively or negatively charged. Then there is the gaseous phase whose composition may deviate from that of the atmosphere above the soil surface. Moreover, there is the aqueous phase which is a dilute aqueous solution, balancing the charges of the solid phase and being strongly buffered by the solid and gaseous phases. For the roots of plants the soil is a resilient provider of nutrients and receiver of excrements. Therefore, for many purposes it is useful to regard the root system itself as an additional phase of the mixture.

Also in application to phase transitions models for porous media stemming from mixture theory are used, e.g., the above-mentioned paper by RICKEN ET AL. [106] on concentration driven phase transitions with application to methane oxidation in landfill cover layers or a paper by BLUHM ET Al. [29] on phase transitions under freezing-thawing load in porous media.

\section{Conclusions and outlook}

It got obvious that a number of scientists dealt with the problem of chemical reactions in mixtures and porous media. In order to formulate a general model, it is necessary to regard the governing rules stemming from thermodynamics. However, there are still several open questions even if the topic is relevant already for a long time and many works to it appeared already fifty years ago. One of this open questions is the temperature: do the constituents of a mixture possess a common temperature or do the constituents have own different temperatures which have to be considered as partial temperatures? Another open question is the exploitation of the second law of thermodynamics. While there is consensus for mixtures of fluids that a couple of methods are applicable, for mixtures containing solids not all methods are accepted. Especially the often used method after COLEMAN and NOLL has been shown not to be entirely correct for solid-fluid mixtures. Nevertheless, applications of this method to such mixtures yielded not totally absurd results. In this case, one can easily circumvent the problems without additional expense in using the method after LIU for which the correctness has been proved also for solid-fluid mixtures. More difficult is the choice of the form of the chemical potential. There exist several suggestions for its introduction and it is by no means clear which of the formulations is correct and yields reasonable results. All the approaches shown in this paper have been introduced, in fact, without verification on a practical example. This is different for the models shown in the last subsection of the paper. These models are all created for a special application, and it was accepted that these models are partly strongly restricted. If one focuses on one special process, this is fair enough, but if one wants to describe several occurrences with one model—as, for example, both the above-mentioned carbonation process and also chemical reactions occurring in reaction walls-it is necessary to formulate it more generally. Thus, in future work a model describing both diffusion and chemical reactions on the basis of the mixture theory will be developed.

Funding Open Access funding enabled and organized by Projekt DEAL.

Open Access This article is licensed under a Creative Commons Attribution 4.0 International License, which permits use, sharing, adaptation, distribution and reproduction in any medium or format, as long as you give appropriate credit to the original author(s) and the source, provide a link to the Creative Commons licence, and indicate if changes were made. The images or other third party material in this article are included in the article's Creative Commons licence, unless indicated otherwise in a credit line to the material. If material is not included in the article's Creative Commons licence and your intended use is not permitted by statutory regulation or exceeds the permitted use, you will need to obtain permission directly from the copyright holder. To view a copy of this licence, visit http://creativecommons.org/licenses/by/4.0/.

\section{References}

1. Albers, B.: Makroskopische Beschreibung von Adsorptions-Diffusions-Vorgängen in porösen Körpern. PhD thesis, TU Berlin, Logos-Verlag, (2000)

2. Albers, B.: Dependence of adsorption/diffusion processes in porous media on bulk and surface permeabilities. Archive Appl. Mech. 53(4-5), 289-306 (2001)

3. Albers, B.: On adsorption and diffusion in porous media. ZAMM 81(10), 683-690 (2001)

4. Albers, B.: Relaxation analysis and linear stability vs. adsorption in porous materials. Continuum Mech. Thermodyn. 151 , 73-95 (2003) 
5. Albers, B.: On results of the surface wave analyses in poroelastic media by means of the Simple Mixture Model and the Biot Model. Soil Dyn. Earthq. Eng. 26, 537-547 (2006)

6. Albers, B.: Analysis of the propagation of sound waves in partially saturated soils by means of a macroscopic linear poroelastic model. Transport Porous Mater. 80(1), 173-192 (2009)

7. Albers, B.: Modeling and Numerical Analysis of Wave Propagation in Saturated and Partially Saturated Porous Media, vol. 48 of Veröffentlichungen des Grundbauinstitutes der Technischen Universität Berlin. Shaker Verlag, Aachen, 2010. Habilitation thesis

8. Albers, B.: On a micro-macro transition for a poroelastic three-component model. ZAMM 90(12), 929-943 (2010)

9. Albers, B.: Main drying and wetting curves of soils: on measurements, prediction and influence on wave propagation. Eng. Trans. 63, 1 (2015)

10. Albers, B.: On the influence of the hysteretic behavior of the capillary pressure on the wave propagation in partially saturated soils. J. Phys.: Conference Series 727 (jun 2016), 012001

11. Albers, B., Wilmanski, K.: On modeling acoustic waves in saturated poroelastic media. J. Engrn. Mech. 131(9), 975-985 (2005)

12. Albers, B., Wilmanski, K.: Continuum Thermodynamics, Part II: Applications and Examples. World Scientific, Singapore (2015)

13. Atkin, R. J., Craine, R. E.: Continuum theories of mixtures. applications. J. Inst. Maths. Applics. 17 (1976)

14. Atkin, R. J., Craine, R. E.: Continuum theories of mixtures. Basic theory and historical development. Quaterly J. Mech. Appl. Math. 29 (1976)

15. Baierlein, R.: The elusive chemical potential. Am. J. Phys. 69(4), 423-434 (2001)

16. Balluffi, R.W., Allen, S.M.: and Carter. Kinetics of Materials. Wiley, W. C. (2005)

17. Bazant, M.Z., Bažant, Z.P.: Theory of sorption hysteresis in nanoporous solids: Part ii molecular condensation. J. Mech. Phys. Solids 60(9), 1660-1675 (2012)

18. Bažant, Z., L'Hermite, R., of Testing, I. U., for Materials, R. L., Structures, and (U.S.), N. S. F.: Mathematical Modeling of Creep and Shrinkage of Concrete. A Wiley Interscience publication. Wiley, 1988

19. Bažant, Z.P., Bazant, M.Z.: Theory of sorption hysteresis in nanoporous solids: Part i: Snap-through instabilities. J. Mech. Phys. Solids 60(9), 1644-1659 (2012)

20. Bear, J.: Dyn. Fluids Porous Media. Dover Publications, New York (1988)

21. Bennethum, L.S., Cushman, J.H.: Multiscale, hybrid mixture theory for swelling systems-I: balance laws. Int. J. Eng. Sci. 34(2), 125-145 (1996)

22. Bennethum, L.S., Cushman, J.H.: Multiscale, hybrid mixture theory for swelling systems-II: constitutive theory. Int. J. Eng. Sci. 34(2), 147-169 (1996)

23. Bennethum, L.S., Murad, M.A., Cushman, J.H.: Clarifying mixture theory and the macroscale chemical potential for porous media. Int. J. Engng. Sci. 34, 1611-1621 (1996)

24. Berryman, J.G.: Confirmation of Biot's theory. Appl. Phys. Lett. 37, 382-384 (1980)

25. Bethke, C.M.: Geochemical Reaction Modeling. Concepts and Applications. Oxford University Press, New York (1996)

26. Biot, M.: Variational-Lagrangian irreversible thermodynamics of initially-stressed solids with thermomolecular diffusion and chemical reactions. J. Mech. Phys. Solids 25(4), 289-307 (1977)

27. Biot, M.A.: General theory of three dimensional consolidation. J. Appl. Phys. 12(2), 155-164 (1941)

28. Biot, M. A.: Theory of propagation of elastic waves in a fluid saturated porous solid, I. low frequency range, II. higher frequency range. J. Acoust. Soc. Am. 28, 2 (1956), 168-178, 179-191

29. Bluhm, J., Bloßfeld, W.M., Ricken, T.: Energetic effects during phase transition under freezing-thawing load in porous media - a continuum multiphase description and FE-simulation. ZAMM - J. Appl. Math. Mech. / Zeitschrift für Angewandte Mathematik und Mechanik 94(7-8), 586-608 (2014)

30. Böhm, M., Devinny, J., Jahani, F., Rosen, G.: On a moving-boundary system modeling corrosion in sewer pipes. Appl. Math. Comput. 92(2), 247-269 (1998)

31. Bowen, R., Wiese, J.: Diffusion in mixtures of elastic materials. Int. J. Eng. Sci. 7, 689-722 (1969)

32. Bowen, R.M.: Toward a thermodynamics and mechanics of mixtures. Arch. Rat. Mech. Anal. 24, 370-403 (1967)

33. Bowen, R.M.: On the stoichiometry of chemically reacting materials. Arch. Rat. Mech. Anal. 29, 114-124 (1968)

34. Bowen, R.M.: The thermochemistry of a reacting mixture of elastic materials with diffusion. Arch. Rat. Mech. Anal. 34, 97-127 (1969)

35. Bowen, R. M.: Theory of mixtures. In Continuum Physics, A. Eringen, Ed., vol. 3. Academic Press, New York, 1976, pp. $2-127$

36. Bowen, R.M.: Incompressible porous media models by use of the theory of mixtures. Int. J. Eng. Sci. 18, 1129-1148 (1980)

37. Bowen, R.M.: Compressible porous media models by use of the theory of mixtures. Int. J. Eng. Sci. 20(6), 697-763 (1982)

38. Bowen, R.M.: Porous media model formulations by the theory of mixtures. In: Bear, J., Corapcioglu, M.Y. (eds.) Fundamentals of Transport Phenomena in Porous Media. Martinus Nijhoff Publishers, Dordrecht (1984)

39. Brunner, F.: Multiphase multicomponent flow in porous media with general reactions: efficient problem formulations, conservative discretizations, and convergence analysis. doctoralthesis, Friedrich-Alexander-Universität Erlangen-Nürnberg (FAU), (2016)

40. Callen, H.B.: Thermodynamics and an Introduction to Thermostatics, 2nd edn. John Wiley and Sons, Hoboken (1985)

41. Chen, L.-Q.: Chemical potential and Gibbs free energy. MRS Bull. 44(7), 520-523 (2019)

42. Coleman, B.D., Noll, W.: The thermodynamics of elastic materials with heat conduction and viscosity. Arch. Rat. Mech. Anal. 13, 167-178 (1963)

43. Cook, G., Dickerson, R.H.: Understanding the chemical potential. Am. J. Phys. 63(8), 737-742 (1995)

44. Coussy, O.: Mech. Porous Continua. John Wiley, Chichester (1995)

45. Creazza, G., Saetta, A., Scotta, R., Vitaliani, R., Onate, E.: Mathematical simulation of structural damage in historical buildings. In Structural studies of historical buildings IV. Volume 1: architectural studies, materials and analysis. Computational Mechanics Publications, Southampton, pp. 111-118 (1995) 
46. de Boer, R., Ehlers, W.: A historical review of the formulation of porous media theories. Acta Mechanica 74, 1-8 (1988)

47. de Groot, S.R., Mazur, P.: Non-Equilibrium Thermodynamics. North-Holland, Amsterdam (1962)

48. Despotou, E., Shtiza, A., Schlegel, T., Verhelst, F.: Literature study on the rate and mechanism of carbonation of lime in mortars. Mauerwerk 20(2), 124-137 (2016)

49. Detmann, B.: On models for porous media containing one, two or three pore fluids and the determination of associated macroscopic material parameters. Mech. Res. Commun. 93, 35-40 (2018)

50. Drdácký, M., Slížková, Z., Ziegenbalg, G.: Nano approach to consolidation of degraded historic lime mortars. J. Nano Res. 8, 13-22 (2009)

51. Dunwoody, N.T.: A thermomechanical theory of diffusion in solid-fluid mixtures. Archive Rational Mech. Anal. 38, 348-371 (1970)

52. Dunwoody, N.T., Müller, I.: Thermodynamic theory of two chemically reacting ideal gases with different temperatures. Arch. Rational Mech. Anal. 29,(1968)

53. Eck, C., Garcke, H., Knabner, P.: Mathematical Modeling. Springer International Publishing, Springer Undergraduate Mathematics Series (2017)

54. Eckart, C.: The thermodynamics of irreversible processes. i. the simple fluid. Phys. Rev. 58, 267-269 (1940)

55. Eringen, A., Ingram, J.D.: A continuum theory of chemically reacting media-I. Int. J. Eng. Sci. 3(2), 197-212 (1965)

56. Fick, A.: Ueber diffusion. Annalen der Physik 170(1), 59-86 (1855)

57. Gatica, J.E., Viljoen, H.J., Hlavacek, V.: Interaction between chemical reaction and natural convection in porous media. Chem. Eng. Sci. 44(9), 1853-1870 (1989)

58. Gawin, D., Pesavento, F., Schrefler, B.A.: Modelling of hygro-thermal behaviour of concrete at high temperature with thermo-chemical and mechanical material degradation. Comput. Methods Appl. Mech. Eng. 192(13), 1731-1771 (2003)

59. Goodman, M., Cowin, S.: A continuum theory of granular materials. Arch. Rat. Mech. Anal. 44, 249-266 (1972)

60. Gray, W.G.: General conservation equations for multi-phase systems: 4 constitutive theory including phase change. Adv. Water Resour. 6(3), 130-140 (1983)

61. Gray, W. G.: Elements of a systematic procedure for the derivation of macroscale conservation equations for multiphase flow in porous media. In Kinetic and Continuum Theories of Granular and Porous Media, K. Hutter and K. Wilmanski, Eds., no. 400 in CISM Courses and Lectures. Springer, Wien-New York, 1999, pp. 67-130

62. Gray, W.G.: Thermodynamics and constitutive theory for multiphase porous-media flow considering internal geometric constraints. Adv. Water Resour. 22(5), 521-547 (1999)

63. Gray, W.G., Hassanizadeh, S.: Macroscale continuum mechanics for multiphase porous-media flow including phases, interfaces, common lines and common points. Adv. Water Resour. 21(4), 261-281 (1998)

64. Gray, W.G., Miller, C.T.: Introduction to the Thermodynamically Constrained Averaging Theory for Porous Medium Systems. Springer, Switzerland (2014)

65. Gray, W.G., Miller, C.T., Schrefler, B.A.: Averaging theory for description of environmental problems: what have we learned, advances. Adv. Water Resour. 51, 123-138 (2013)

66. Green, A.E., Naghdi, P.M.: A dynamical theory of interacting continua. Int. J. Engng. Sci. 3, 231-241 (1965)

67. Gurtin, M.E., Fried, E., Anand, L.: The Mechanics and Thermodynamics of Continua. Cambridge University Press, New York (2010)

68. Hassanizadeh, M., Gray, W.G.: General conservation equations for multi-phase systems: 1. averaging procedure. Adv. Water Resour. 2, 131-144 (1979)

69. Hassanizadeh, M., Gray, W.G.: General conservation equations for multi-phase systems: 2. mass, momenta, energy, and entropy equations. Adv. Water Resour. 2, 191-203 (1979)

70. Hassanizadeh, M., Gray, W.G.: General conservation equations for multi-phase systems: 3. constitutive theory for porous media flow. Adv. Water Resour. 3(1), 25-40 (1980)

71. Hassanizadeh, S.M., Gray, W.G.: Mechanics and thermodynamics of multiphase flow in porous media including interphase boundaries. Adv. Water Resour. 13(4), 169-186 (1990)

72. Helgeson, H.C.: Evaluation of irreversible reactions in geochemical processes involving minerals and aqueous solutions I. thermodynamic relations. Geochimica et Cosmochimica Acta 32(8), 853-877 (1968)

73. Helgeson, H.C., Brown, T.H., Nigrini, A., Jones, T.A.: Calculation of mass transfer in geochemical processes involving aqueous solutions. Geochimica et Cosmochimica Acta 34(5), 569-592 (1970)

74. Helgeson, H.C., Murphy, W.M.: Calculation of mass transfer among minerals and aqueous solutions as a function of time and surface area in geochemical processes. I. computational approach. J. Int. Assoc. Math. Geol. 15, 109-130 (1983)

75. Hoffmann, J., Kräutle, S., Knabner, P.: A general reduction scheme for reactive transport in porous media. Comput. Geosci. 16, 1081-1099 (2012)

76. Hornung, U.: Homogenization and Porous Media. Interdisciplinary Applied Mathematics Series. Springer, New-York (1997)

77. Houst, Y.F., Wittmann, F.H.: Influence of porosity and water content on the diffusivity of co2 and o 2 through hydrated cement paste. Cement Concrete Res. 24(6), 1165-1176 (1994)

78. Houst, Y.F., Wittmann, F.H.: Depth profiles of carbonates formed during natural carbonation. Cement Concrete Res. 32(12), 1923-1930 (2002)

79. Hütter, G.: Coleman-noll procedure for classical and generalized continuum theories. In: Altenbach, H., Öchsner, A. (eds.) Encyclopedia of Continuum Mechanics, pp. 1-8. Springer, Berlin Heidelberg (2017)

80. Hutter, K., Jöhnk, K.: Continuum Methods of Physical Modeling. Turbulence. Springer, Berlin, Continuum Mechanics, Dimensional Analysis (2004)

81. Ingram, J.D., Cemal Eringen, A.: A continuum theory of chemically reacting media-II Constitutive equations of reacting fluid mixtures. Int. J. Eng. Sci. 5(4), 289-322 (1967)

82. Job, G., Herrmann, F.: Chemical potential-a quantity in search of recognition. Euro. J. Phys. 27(2), 353-371 (2006)

83. Job, G., Rüffler, R.: Physical Chemistry from a Different Angle. Springer, Berlin (2016)

84. Kenyon, D.E.: Thermostatics of solid-fluid mixtures. Archive Rational Mech. Anal. 62(2), 117-129 (1976) 
85. Kristensson, O.: Extrapolation of the models developed to the repository long term evolution and evaluation of uncertainties - Review of thermomechanical continuum mixture theories applicable for EBS materials. Deliverable-No.: D3.5-4a of the Project Long-term Performance of Engineered Barrier Systems PEBS co-funded by the European Commission, downloadable

86. Lichtner, P.: Continuum formulation of multicomponent-multiphase reactive transport. Rev. Mineral. 34, 1-81 (1996)

87. Lichtner, P.C.: Continuum model for simultaneous chemical reactions and mass transport in hydrothermal systems. Geochimica et Cosmochimica Acta 49(3), 779-800 (1985)

88. Lichtner, P.C., Steefel, C.I., Oelkers, E.H.: Reactive Transport in Porous Media. Reviews in Mineralogy, vol. 34. The Mineralogical Society of America, Washington (1996)

89. Liu, I.-S.: Method of lagrange multipliers for exploitation of the entropy principle. Arch. Rat. Mech. Anal. 46, 131-148 (1972)

90. Marle, C.: On macroscopic equations governing multiphase flow with diffusion and chemical reactions in porous media. Int. J. Eng. Sci. 20(5), 643-662 (1982)

91. Müller, I.: A thermodynamic theory of mixtures of fluids. Archive Rational Mech. Anal. 28, 1-39 (1968)

92. Müller, I.: Die Kältefunktion, eine universelle Funktion in der Thermodynamik viskoser wärmeleitender Flüssigkeit. Arch. Rat. Mech. Anal. 40, 1-36 (1971)

93. Müller, I.: Thermodynamics. Pitman, N.Y. (1985)

94. Muntean, A., Böhm, M., Kropp, J.: Moving carbonation fronts in concrete: a moving-sharp-interface approach. Chem. Eng. Sci. 66(3), 538-547 (2011)

95. Naidu, R., Birke, V. (eds.): Permeable Reactive Barrier. Sustainable Groundwater Remediation. CRC Press, Boca Raton (2015)

96. Noll, W.: Theory of interpenetrating continuous bodies. In: Proceedings of EUROMECH Colloquium 290 on Mechanics of Swelling, Rhodes, Greece, August 1993 (1993), A. S. T.K. Karalis, Ed

97. Nunziato, J.W., Walsh, E.K.: On ideal multiphase mixtures with chemical reactions and diffusion. Arch. Rat. Mech. Anal. 73, 285-311 (1980)

98. Passman, S.L.: Mixtures of granular materials. Int. J. Eng. Sci. 15, 117-129 (1977)

99. Passman, S. L., Nunziato, J. W., Walsh, E. K.: A theory of multiphase mixtures. In: Truessdell's Rational Thermodynamics, C. Truesdell, Ed., vol. App. 5C. Springer, 1984, pp. 286-325

100. Pecker, C., Deresiewicz, H.: Thermal effects on wave propagation in liquid-filled porous media. Acta Mechanica 16, 45-64 (1973)

101. Plona, T.J.: Observation of a second bulk compressional wave in a porous medium at ultrasonic frequencies. Appl. Phys. Lett. 36(4), 259-261 (1980)

102. Prigogine, I.: Introduction to Thermodynamics of Irreversible Processes. Interscience, New York (1967)

103. Raats, P.A.C.: Applications of the theory of mixtures in soil physics. In: Truesdell, C.A. (ed.) Rational Thermodynamics. Springer, New York (1984)

104. Rajagopal, K. R., Tao, L.: Mechanics of Mixtures. World Scientific, Singapore (1995)

105. Rice, J.R., P., C. M. : Some basic stress-diffusion solutions for fluid saturated elastic porous media with compressible constituents. Rev. Geophys. Space Phys. 14, 227-241 (1976)

106. Ricken, T., Sindern, A., Bluhm, J., Widmann, R., Denecke, M., Gehrke, T., Schmidt, T.: Concentration driven phase transitions in multiphase porous media with application to methane oxidation in landfill cover layers. ZAMM - J. Appl. Math. Mech./Zeitschrift für Angewandte Mathematik und Mechanik 94(7-8), 609-622 (2014)

107. Roques, H., Girou, A.: Kinetics of the formation conditions of carbonate tartars. Water Res. 8(11), 907-920 (1974)

108. Saetta, A.V., Schrefler, B.A., Vitaliani, R.V.: The carbonation of concrete and the mechanism of moisture, heat and carbon dioxide flow through porous materials. Cement Concrete Res. 23(4), 761-772 (1993)

109. Saetta, A.V., Schrefler, B.A., Vitaliani, R.V.: 2 - D model for carbonation and moisture/heat flow in porous materials. Cement Concrete Res. 25(8), 1703-1712 (1995)

110. Sahimi, M.: Flow and Transport in Porous Media and Fractured Rock, from Classical Methods to Modern Approaches. Wiley-VCH, Weinheim (2011)

111. Schrefler, B. A.: Numerical methods in theories of porous materials. In: Kinetic and Continuum Theories of Granular and Porous Media, H. K. and W. K., Eds., vol. 400 of CISM Lecture Notes. Springer, Wien, 1999, pp. 187-244

112. Schrefler, B.A., Simoni, L., Majorana, C.E.: A general model for the mechanics of saturated-unsaturated porous materials. Materials Struct 22, 323-334 (1989)

113. Schroeder, D. V.: An introduction to thermal physics. Addison-Wesley, San Francisco [u.a.], (2000)

114. Silhavy, M.: The Mechanics and Thermodynamics of Continuous Media. Springer, Berlin (1997)

115. Slattery, J.C., Sagis, L., Oh, E.-S.: Interfacial Transport Phenomena. Springer, New York (2013)

116. Steefel, C.I., DePaolo, D.J., Lichtner, P.C.: Reactive transport modeling: an essential tool and a new research approach for the earth sciences. Earth Planetary Sci. Letts. 240(3), 539-558 (2005)

117. Stefan, J.: Über das Gleichgewicht und die Bewegung, insbesondere die Diffusion von Gasgemengen. Sitzungsberichte der Mathematisch-Naturwissenschaftlichen Classe der Kaiserlichen Akademie der Wissenschaften Wien, 2te Abteilung 63 (1871), 63-124

118. Triani, V., Papenfuss, C., Cimmelli, V.A., Muschik, W.: Exploitation of the second law: Coleman-Noll and Liu procedure in comparison. J Non-Equilibrium Thermodyn 33(1), 47-60 (2008)

119. Truesdell, C.A.: Rational Thermodynamics (Second Edition). Springer, Berlin (1984)

120. Truesdell, C. A., Toupin, R. A.: The Classical Field Theories, vol. III/1. Springer, Berlin, 1960. Flügge, S. (ed.)

121. van Balen, K., van Gemert, D.: Modelling lime mortar carbonation. Mater. Struct. 27, 393-398 (1994)

122. Veldsink, J.W., van Damme, R.M.J., Versteeg, G.F., van Swaaij, W.P.M.: The use of the dusty-gas model for the description of mass transport with chemical reaction in porous media. Chem. Eng. J. Biochem. Eng. J. 57(2), 115-125 (1995)

123. Vromans, A.J., Muntean, A., van de Ven, A.A.F.: A mixture theory-based concrete corrosion model coupling chemical reactions, diffusion and mechanics. Pacific J. Math. Ind. 10, 5 (2018) 
124. Williams, W.O.: Constitutive equations for flow in an incompressible viscous fluid through a porous medium. Quarterly Appl. Math. 36(3), 255-267 (1978)

125. Wilmanski, K.: Lagrangean model of two-phase porous material. J. Non-Equilibrium Thermodyn. 20, 50-77 (1995)

126. Wilmanski, K.: Porous media at finite strains - the new model with the balance equation for porosity. Arch. Mech. 48(4), 591-628 (1996)

127. Wilmanski, K.: Thermomechanics of Continua. Springer, Berlin, N.Y. (1998)

128. Wilmanski, K.: Thermodynamical admissibility of Biot's model of poroelastic saturated materials. Archives Mech. 54(5-6), 709-736 (2002)

129. Wilmanski, K.: A few remarks on micro/macro transitions and Gassmann relations for poroelastic materials. Mech. Solids Struct. 6, 191-204 (2007)

130. Wilmanski, K.: Continuum Thermodynamics, Part I: Foundations. World Scientific, Singapore (2008)

131. Woods, L.C.: The Thermodynamics of Fluid Systems. Clarendon Press, Oxford engineering science series (1975)

132. Zouridakis, N.M., Economou, I.G., Tzevelekos, K.P., Kikkinides, E.S.: Investigation of the physicochemical characteristics of ancient mortars by static and dynamic studies. Cement Concrete Res. 30(7), 1151-1155 (2000)

Publisher's Note Springer Nature remains neutral with regard to jurisdictional claims in published maps and institutional affiliations. 